\title{
Herculanum, architecture domestique et décor. Restitution graphique et virtuelle de la casa di Nettuno ed Anfitrite (V, 6-7). Campagne $2017 \mathrm{du}$ programme ANR VESUVIA
}

Alexandra Dardenay, James N. Andrews, Hélène Eristov, Marie-Laure Maraval and Nicolas Monteix

\section{(2) OpenEdition \\ Journals}

Electronic version

URL: http://journals.openedition.org/cefr/2119

DOI: $10.4000 /$ cefr. 2119

ISSN: 2282-5703

Publisher

École française de Rome

\section{Electronic reference}

Alexandra Dardenay, James N. Andrews, Hélène Eristov, Marie-Laure Maraval and Nicolas Monteix, " Herculanum, architecture domestique et décor. Restitution graphique et virtuelle de la casa di Nettuno ed Anfitrite (V, 6-7). Campagne 2017 du programme ANR VESUVIA », Chronique des activités archéologiques de l'École française de Rome [Online], Les cités vésuviennes, Online since 14 February 2019, connection on 01 May 2019. URL : http://journals.openedition.org/cefr/2119; DOI : 10.4000/ cefr.2119

This text was automatically generated on 1 May 2019.

(c) École française de Rome 


\title{
Herculanum, architecture
} domestique et décor. Restitution graphique et virtuelle de la casa di Nettuno ed Anfitrite (V, 6-7). Campagne $2017 \mathrm{du}$ programme ANR VESUVIA

\author{
Alexandra Dardenay, James N. Andrews, Hélène Eristov, Marie-Laure \\ Maraval and Nicolas Monteix
}

Notre reconnaissance la plus sincère est adressée aux institutions mentionnées en début de cet article et qui apportent leur collaboration au projet VESUVIA.

1 Au cours des missions menées en 2017, les décors peints de plusieurs espaces de la casa di Nettuno ed Anfitrite ont fait l'objet de restitutions infographiques (fig. 1). Ces infographies ont ensuite été insérées dans le modèle 3D, qui sert de support à l'étude architecturale et ornementale globale de cette domus de l'insula $\mathrm{V}$ d'Herculanum. Par ailleurs, en étendant notre étude aux étages, il est possible d'envisager ici l'analyse de l'édifice dans son ensemble, dans une perspective diachronique ${ }^{1}$.

Fig. 1 - Casa di Nettuno ed Anfitrite. Plans du rez-de-chaussée et de l'étage avec les pièces restituées. 

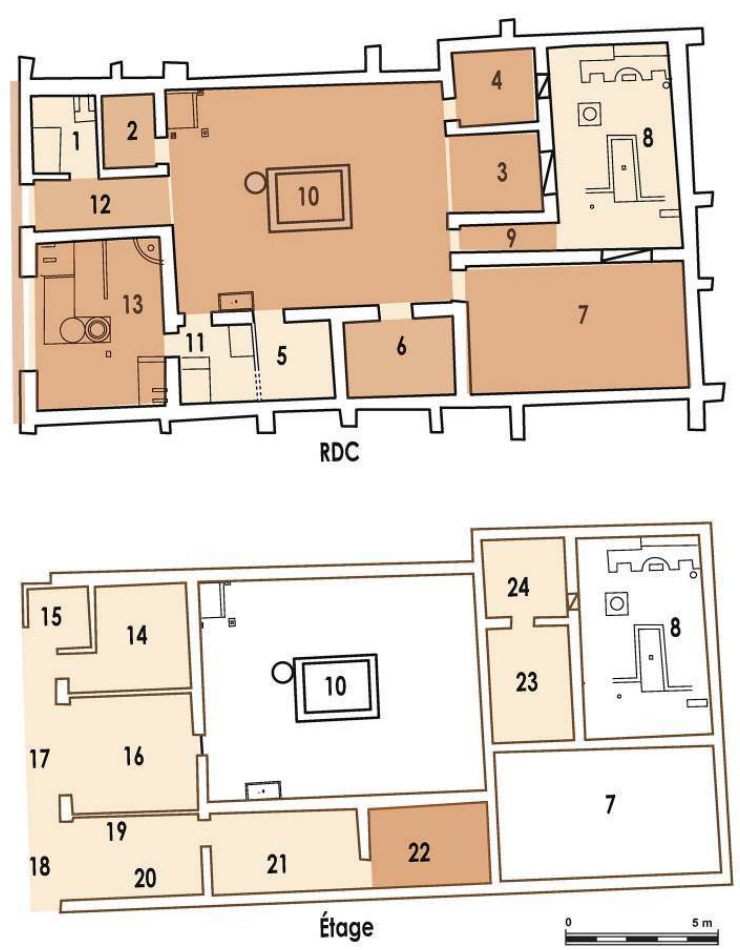

M.-L. Maraval, N. Monteix, ANR Vesuvia - 2018. Licence creative commons, (c) CC BY-NC-ND.

La question de l'étude des pavements a également été envisagée. La parution récente du Corpus des mosaïques d'Herculanum ${ }^{2}$ peut servir de base à cette analyse. Malheureusement, ce volume ne propose pas de datation archéologique des pavements, mais des datations stylistiques, fondées d'une part sur la chronologie traditionnelle des pavements romains, et d'autre part, sur celle des peintures murales de chaque espace concerné. Les datations des pavements d'Herculanum sont ainsi mentionnées en «styles pompéiens $»^{3}$. La fragilité de cette démarche est avérée, car la datation des pavements d'après la chronologie des peintures murales nécessiterait la mise en œuvre de l'étude archéologique du lien mur/sol. Or à Herculanum cette entreprise est, sans doute, vaine. En effet, toutes les peintures d'Herculanum ont été détachées des parois pendant les fouilles d'A. Maiuri, restaurées, et replacées sur les murs, avec parfois des décalages nettement perceptibles. Autrement dit, aucune peinture d'Herculanum n'est archéologiquement in situ. Toutes les jonctions ayant donc été détruites, l'étude des liens murs/sols doit être menée avec prudence, en s'appuyant autant que possible sur les résultats de fouilles, quand ils existent.

\section{Restitution des décors peints du rez-de-chaussée}

\section{Fauces 12}

Les deux murs des fauces $12(4,60 \mathrm{~m} \times 1,64 \mathrm{~m})$ sont de longueur inégale $(4,80 \mathrm{~m}$ au sud; $3,90 \mathrm{~m}$ au nord) en raison de l'obliquité du mur de façade par rapport à l'axe de ce couloir d'accès (fig. 2). Compte tenu de l'épaisseur des chambranles vers la façade $(0,25 \mathrm{~m})$ et 
vers l'atrium $(0,41 \mathrm{~m})$, la longueur du décor est de 4,17 $\mathrm{m}$ au sud et de 3,26 $\mathrm{m}$ au nord. La hauteur moyenne du décor, conservée sur 2,67 m, devait culminer à 2,72 $\mathrm{m}^{4}$.

Fig. 2 - Casa di Nettuno ed Anfitrite. Fauces 12, mur nord. Orthophotographie.
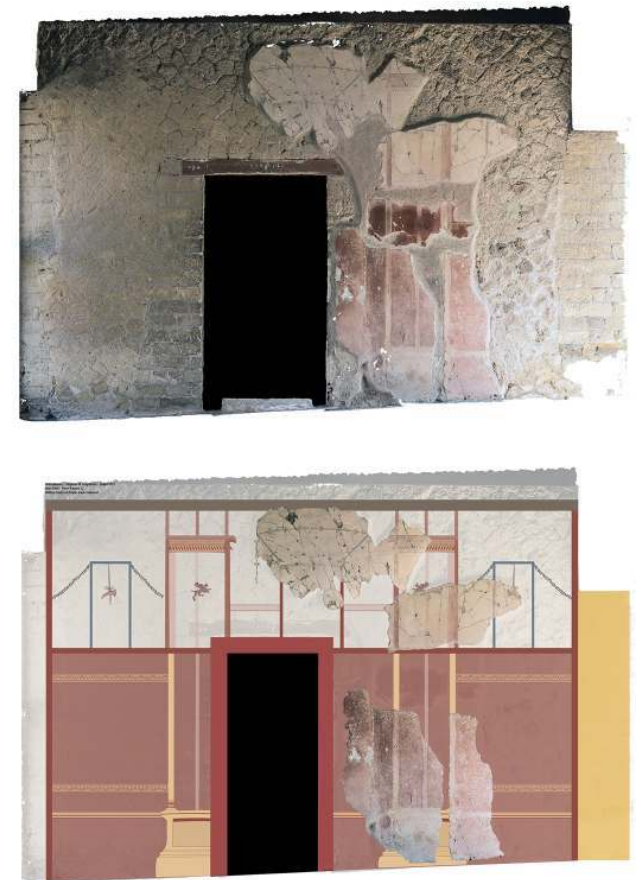

Mibact, Archeovision, M.-L. Maraval, H. Eristov, ANR Vesuvia - 2018. Licence creative commons, (c) CC BY-NC-ND.

4 Des éléments suffisants sur les trois zones de la paroi assurent la restitution du schéma décoratif et de la plupart de ses détails. Sur le mur nord, le mieux conservé, il reste environ un tiers du décor au centre et à droite, du soubassement jusqu'à la zone supérieure comprise. Sur le tiers droit du mur sud, subsistent un fragment de soubassement et d'amorce de la zone médiane ainsi qu'un fragment du haut de la zone médiane et d'une partie de la zone supérieure.

déma décoratif superpose une zone basse rouge scandée par de gros piédestaux et couronnée par une moulure fictive, une zone médiane rouge articulée par des supports reposant sur les piédestaux et délimitant des panneaux à bordures ajourées, une moulure fictive puis une zone supérieure à fond blanc et architectures légères.

6 La hauteur des zones est assurée : 1,18 m (moulures non comprises) pour la zone médiane, et $1,07 \mathrm{~m}$ (moulures non comprises) pour la zone supérieure. En raison de la pente ${ }^{5}$, la hauteur du soubassement varie de 0,51 m à l'ouest, à 0,36 m à l'est (mur nord) et de 0,45 m à l'ouest à $0,27 \mathrm{~m}$ à l'est (mur sud).

7 En zone basse, sans plinthe, les gros piédestaux jaunes sont attestés à l'extrémité ouest du mur sud et à droite du mur nord où l'on distingue un profil en talon ; il est possible que le corps du piédestal jaune ait été traité en longues feuilles comme dans la pièce 4 , mais l'état d'érosion ne permet pas de l'affirmer. L'absence de plinthe a amené les décorateurs à suivre la pente du sol, de sorte que la base des piédestaux est oblique et que leur 
hauteur varie, de 0,41 m à droite du mur nord, à 0,49 m à gauche. En couronnement de la zone basse, la moulure fictive (visible sur le mur nord) superpose, sur une épaisseur de $4 \mathrm{~cm}$, des bandes ocre jaune en dégradé.

Comme on l'a déjà remarqué dans d'autres pièces (triclinium 7 et atrium, mur sud) le positionnement de certaines plaques lors des fouilles pose problème. Sur le mur sud des fauces, une plaque de soubassement a été repositionnée à l'extrémité droite (ouest). Or le dénivelé ne le permet pas : étant donné que le côté ouest qui descend vers la rue offre la plus grande hauteur de soubassement, la moulure fictive se trouverait, du côté est, à $0,15 \mathrm{~m} \mathrm{du}$ sol, alors que sur le mur nord, au même endroit, elle se trouve à $0,30 \mathrm{~m}$. Il faut donc déplacer ce fragment. Puisqu'il a conservé, à son extrémité gauche, une amorce de piédestal jaune, celui-ci correspond à l'architecture de gauche, et le fragment ne peut se situer qu'au centre de la paroi. Dans ce cas la moulure se trouve, au centre du mur, à $0,30 \mathrm{~m} \mathrm{du}$ sol, alors que sur le mur nord au même endroit elle est à $0,40 \mathrm{~m}$. Il est probable que la pente du sol, mal contrôlée par les décorateurs, a causé ces irrégularités.

9 La zone médiane comporte trois panneaux séparés par des architectures schématiques : sur le mur nord, des paires de colonnes (h. $1,15 \mathrm{~m})$ reposent sur les piédestaux; les moulures de leur base sont rendues par trois à cinq traits bordeaux, sans que l'on sache si cette disparité entre la colonne de droite et celle de gauche révèle une quelconque différence de statut. Toute trace de chapiteau a disparu. Entre les colonnes, au centre de cette architecture, une bande verticale se prolonge en zone supérieure. Sur le mur sud, le traitement de ces supports diffère; les architectures sont encadrées de bandes bleues entre des filets blancs; la fonction architecturale des piédestaux s'affaiblit puisqu'ils ne portent qu'une colonne qui redouble le cadre vers les champs latéraux.

10 En bas du champ droit du mur nord, une bordure ajourée d'exécution très relâchée comporte une alternance de triangles et d'arceaux auxquels s'ajoutent, dans tous les intervalles, des trifols ; un double filet encadre la bordure vers l'intérieur du panneau, un filet simple vers l'extérieur.

11 Ici aussi, le positionnement d'une plaque est fautif : un fragment inséré à droite du mur nord entre la zone médiane et la zone supérieure n'est pas cohérent avec cette paroi car il comporte à gauche non une colonne, mais une bande bleue. Il s'ajoute une difficulté supplémentaire due à la présence d'une bordure ajourée à triangles et arceaux mais dont le double filet est orienté vers l'extérieur du panneau et non vers l'intérieur. La seule solution satisfaisante consiste non seulement à déplacer le fragment sur le mur sud, mais encore à le retourner et à le positionner en bas de l'architecture de gauche, de sorte que la bande bleue se situe, comme son symétrique, en bordure du champ central, et que la bordure ajourée se trouve en bas du panneau gauche avec son double filet correctement placé.

Dans la proposition de restitution, une bordure ajourée est supposée en haut des panneaux latéraux, symétrique à celle du bas.

13 La zone supérieure, bien attestée par les fragments du mur nord, s'organise autour d'un pseudo édicule schématique réduit à un simple encadrement rouge légèrement cintré muni de petites volutes en acrotères d'où partent des tiges obliques soutenant une guirlande qui redouble la structure du pseudo-édicule. À mi-hauteur, des bordures horizontales (sans doute ajourées mais qui ont perdu toute trace de leur décor) le relient à des pavillons latéraux alignés sur les paires de colonnes de la zone médiane. Plafond à solives et entablement reposent sur des supports peu détaillés et abritent un animal dont 
subsistent les pattes arrière, l'une tendue, l'autre très repliée ; sur le modèle de l'un des fauves du couloir 9, il est identifié comme un griffon en vol tourné vers le centre de la paroi. Étant donné que sur le fragment du mur sud apparaissent pattes et ailes, un griffon identique est restitué sur les deux murs. Un second niveau très schématique surmonte ces pavillons et se réduit à trois bandes verticales, deux d'entre elles alignées sur les supports du premier niveau, la troisième passant, étrangement, au centre du pavillon et derrière le griffon. Aux extrémités de la paroi, le fond blanc est occupé, en guise d'architecture, par un encadrement linéaire bleu d'où pend un rhyton suspendu à des rubans formant un nœud à coques, et que de fines guirlandes relient, d'un côté au pavillon, de l'autre à l'angle du mur. Sachant que le rhyton se trouve au centre du compartiment bleu, sa largeur est assurée, et sa hauteur se fonde sur le prolongement de la courbe de la guirlande ; il s'aligne, ainsi, sur le pseudo-édicule central. Une large bande horizontale gris foncé $(5 \mathrm{~cm})$ forme la limite avec le plafond.

Techniquement, la trace d'une journée de travail recouverte par une bande rouge effacée a pu être repérée à la base de la zone supérieure, sur le mur sud comme sur le mur nord.

Le pavement en béton de tuileau, très grossier, des fauces et de l'atrium est daté du IV ${ }^{e}$ style par les auteurs du Corpus des mosaïques d'Herculanum ${ }^{6}$. La datation repose sur un double argument: d'une part, les auteurs remarquent que la présence d'inclusions de marbres polychrome dans le pavement de « cementizio a base fittile (cocciopesto) » n'est pas documentée en contexte privé avant la fin du $\mathrm{I}^{\mathrm{er}}$ siècle avant notre ère. D'autre part, ils observent que les peintures murales de cet espace (tout comme celui de l'atrium, et de la plupart des pièces de la maison) furent refaites dans les dernières années du site. Ils estiment dès lors que le pavement peut être daté du IV style, tout comme les enduits peints. Certes, les pavements peuvent être contemporains de la dernière phase de réfection de la maison, tout comme ils peuvent être bien plus anciens, et dater de la phase augustéenne correspondant à la reconstruction de la maison et à un premier état de décor peint attesté. D'autant que durant les dernières années avant l'éruption, cette maison faisait l'objet d'une ambitieuse rénovation (peintures scénographiques, installation d'un laraire entièrement recouvert de marbre, aménagement d'un nymphée orné de mosaïques pariétales, etc.) qui n'était pas achevée, notamment dans l'atrium, attendant de voir ses enduits peints terminés ${ }^{7}$. Ne peut-on supposer dès lors que le grossier pavement de mortier qui se trouvait dans les fauces et l'atrium était destiné à être recouvert d'un sol neuf, après l'achèvement des peintures ? La question mérite, quoi qu'il en soit, d'être posée. De plus, d'après les auteurs, le pavement $\mathrm{III}^{\mathrm{e}}$ style de l'oecus 20 la casa d'Argo confirme la datation $\mathrm{IV}^{\mathrm{e}}$ style du pavement de l'atrium et des fauces de la casa di Nettuno ed Anfitrite ${ }^{8}$. On voit comme ces datations stylistiques sont fragiles, et les raisonnements circulaires.

\section{Pièce 2}

16 Cette petite pièce s'ouvre sur la partie occidentale de l'atrium, à proximité du laraire maçonné $(1,64 \mathrm{~m} \times 2,43 \mathrm{~m}$; fig. 3). Sur la paroi en pan de bois hourdé (agraticcio), moderne, les lambeaux d'enduits conservent tous les éléments d'un système simple à fond blanc et décor linéaire ocre jaune, dénué de soubassement et dont la zone supérieure est mal circonscrite. Les parois délimitées par de larges bandes rouges aux angles s'articulent en panneaux blancs (à $0,48 \mathrm{~m}$ du sol) encadrés par un feston ocre jaune ponctué de fleurons et que redouble un large cadre rouge. L'exécution est très relâchée et 
les festons de largeur et de courbure irrégulières. Quelques variantes différencient les quatre murs de la pièce.

Fig. 3 - Casa di Nettuno ed Anfitrite. Pièce 2, murs ouest et nord.

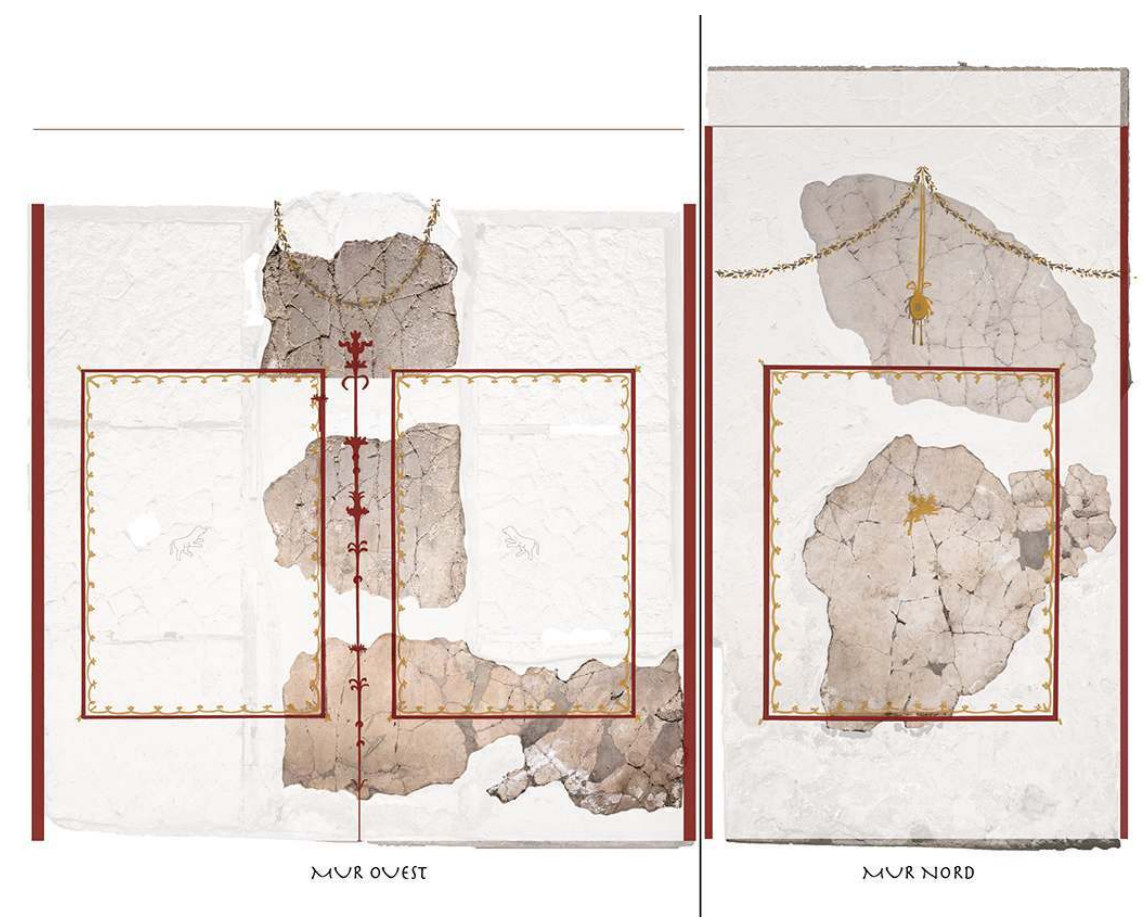

Mibact, M.-L. Maraval, H. Eristov, ANR Vesuvia - 2018. Licence creative commons, (c) CC BY-NC-ND

Le système décoratif est lisible sur le mur est : à gauche de la porte donnant sur l'atrium, un panneau haut de 1,31 m et large de 0,89 m, ne semble pas avoir comporté de vignette. On note que le bas du panneau se situe à $0,33 \mathrm{~m}$ du sol, en net décalage par rapport aux autres murs : de fait, partout le manque de soin se révèle dans le traitement des angles où les fleurons se réduisent à une tache de pinceau; on note toutefois une meilleure exécution des festons du côté droit du panneau. À droite de la porte, la largeur très réduite ne permet d'installer que deux bandes rouges verticales, l'une bordant l'angle de la pièce, l'autre la limite droite de la porte. La largeur de l'embrasure de la porte est traitée de la même façon, avec deux bandes rouges.

Du mur ouest que n'interrompt aucune ouverture, sont conservés les angles inférieurs de deux panneaux à festons séparés par un candélabre schématique à ombelles, crochets et bagues. Son sommet est ponctué d'un monstre schématique à deux ailes et "tête" couronnée. Au-dessus, le champ blanc de la zone supérieure s'orne d'une guirlande verte en demi-cercle qui n'occupe que la portion centrale du mur et dont le mode d'accrochage à la limite du plafond n'est pas conservé.

Sur le mur nord, le panneau, large de 1,11 m, porte un griffon en vol tourné vers la droite, assez bien conservé : on distingue le bec d'oiseau, deux cornes, deux grandes ailes, une patte arrière tendue, l'autre fortement repliée. Au-dessus, dans le champ blanc de la zone supérieure, un tambourin est suspendu entre deux guirlandes accrochées aux bandes verticales rouges des angles de la paroi et, au centre, à la limite avec le plafond. Le mur sud n'a conservé son enduit qu'à l'angle inférieur droit. Le décor restitué est identique à celui du mur nord. 
20 Dans cette petite pièce, les arbitrages de restitution, peu nombreux, concernent les animaux en vol, schématiquement rendus au trait, identiques à celui du mur nord : sur les deux panneaux du mur ouest, on les suppose affrontés, et, sur le mur sud, le griffon est positionné symétriquement par rapport à celui du mur nord. En réalité, ces vignettes représentaient très probablement des animaux ou des monstres divers, et non un motif partout identique ; mais en l'absence de vestige, le choix s'est porté sur la répétition de la seule forme attestée dans la pièce. Sur le mur est, en revanche, il ne semble pas y avoir eu de vignette au centre du panneau.

21 La seconde inconnue touche au mode d'accrochage des guirlandes, tant sur les bandes rouges verticales qu'à la limite avec le plafond : toute trace de filet ou de compartiment ayant disparu en zone haute, les deux guirlandes sont prolongées jusqu'aux limites du mur.

Enfin la zone haute du mur est pose problème : le système du mur ouest à deux panneaux séparés par un candélabre que surmonte une guirlande ne peut se reproduire à l'est en raison de la présence de la porte, de sorte qu'il faudrait reprendre le double feston des murs nord et sud; mais le décalage de hauteur du panneau médian (situé $0,15 \mathrm{~m}$ plus bas que sur les autres murs) amènerait à étirer démesurément le motif. Dans le doute, cette zone haute a donc été laissée vide.

Ce type de décor propre aux « Nebenzimmer ${ }^{9}$ » se retrouve, par exemple, à Pompéi, dans la casa dei quattro stili $(\mathrm{I} 8,17)$, dans les pièces [14] et [23].

Le sol de cette pièce n'a pu être documenté.

\section{Pièce 6}

Donnant sur le côté sud de l'atrium, cette pièce de 2,33 $\mathrm{m} \times 2,50 \mathrm{~m}$ a perdu presque tout son décor (fig. 4). Il reste, à droite du mur sud, un lambeau du soubassement, au centre du mur ouest, un fragment du soubassement et un autre du haut de la zone médiane; quant aux enduits peints des murs nord et est, ils ont presque totalement disparu: du mur nord, subsistent le tiers gauche du soubassement et un lambeau à droite de la porte, et du mur est aucun vestige. 
Fig. 4 - Casa di Nettuno ed Anfitrite. Pièce 6, murs ouest et nord.

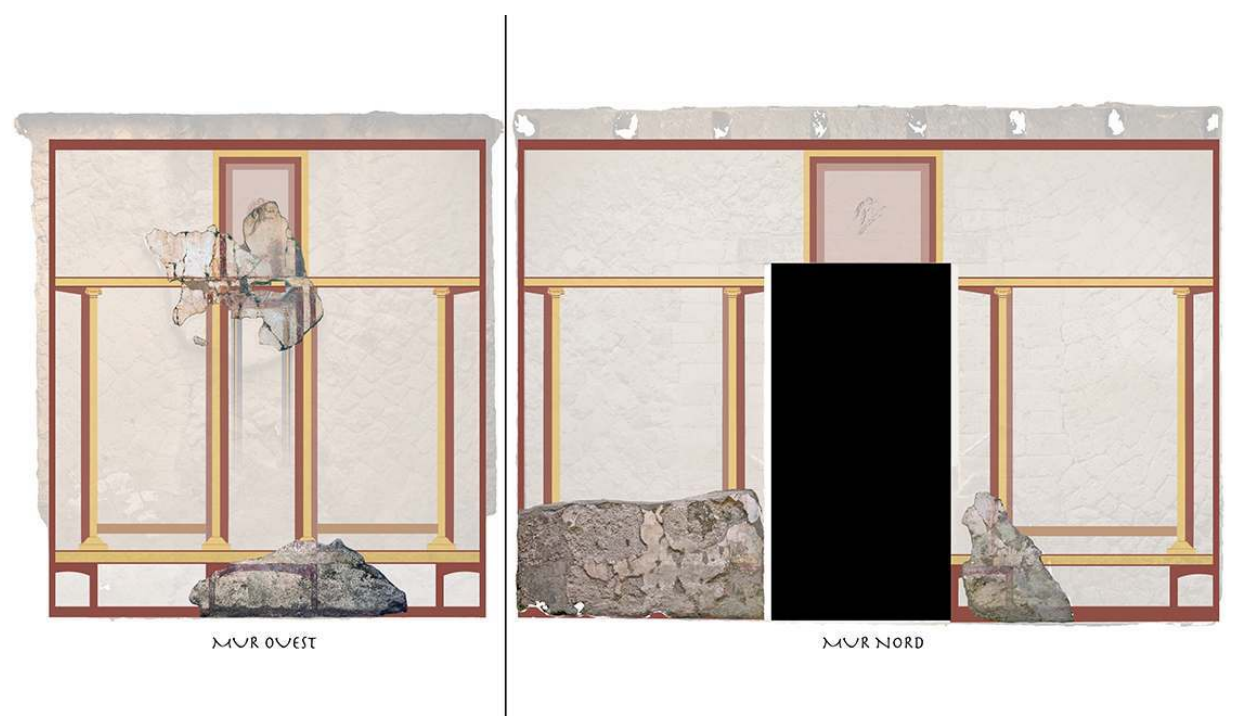

Mibact, M.-L. Maraval, H. Eristov, ANR Vesuvia - 2018. Licence creative commons, (c) CC BY-NC-ND. du mur est: au-dessus d'un étroit compartiment linéaire rouge en zone basse, une architecture se superpose. Sa restitution s'appuie sur celle des longs murs nord et sud. Sur le mur sud, non interrompu par la porte, la structure à architecture centrale et étroites architectures latérales est assurée par la présence d'une base de colonne jaune à l'extrémité droite du fragment insitu. Cette donnée témoigne de la largeur de l'architecture latérale $(0,28 \mathrm{~m})$. Sur le mur nord, les fragments en partie basse de part et d'autre de la porte vers l'atrium impliquent les mêmes dimensions de champs latéraux $(1,07 \mathrm{~m})$ que sur le mur ouest. l'intérieur d'une série de bandes ocre jaune, rouge, violacé, subsiste la moitié inférieure d'un corps de harpie en vol, tournée vers la droite, tenant une flûte dans chaque main ( fig. 5). En l'absence de traces sur les autres murs, une forme linéaire de même type est restituée en zone supérieure des parois est, nord, sud. 
Fig. 5 - Casa di Nettuno ed Anfitrite. Pièce 6, mur ouest, harpie.

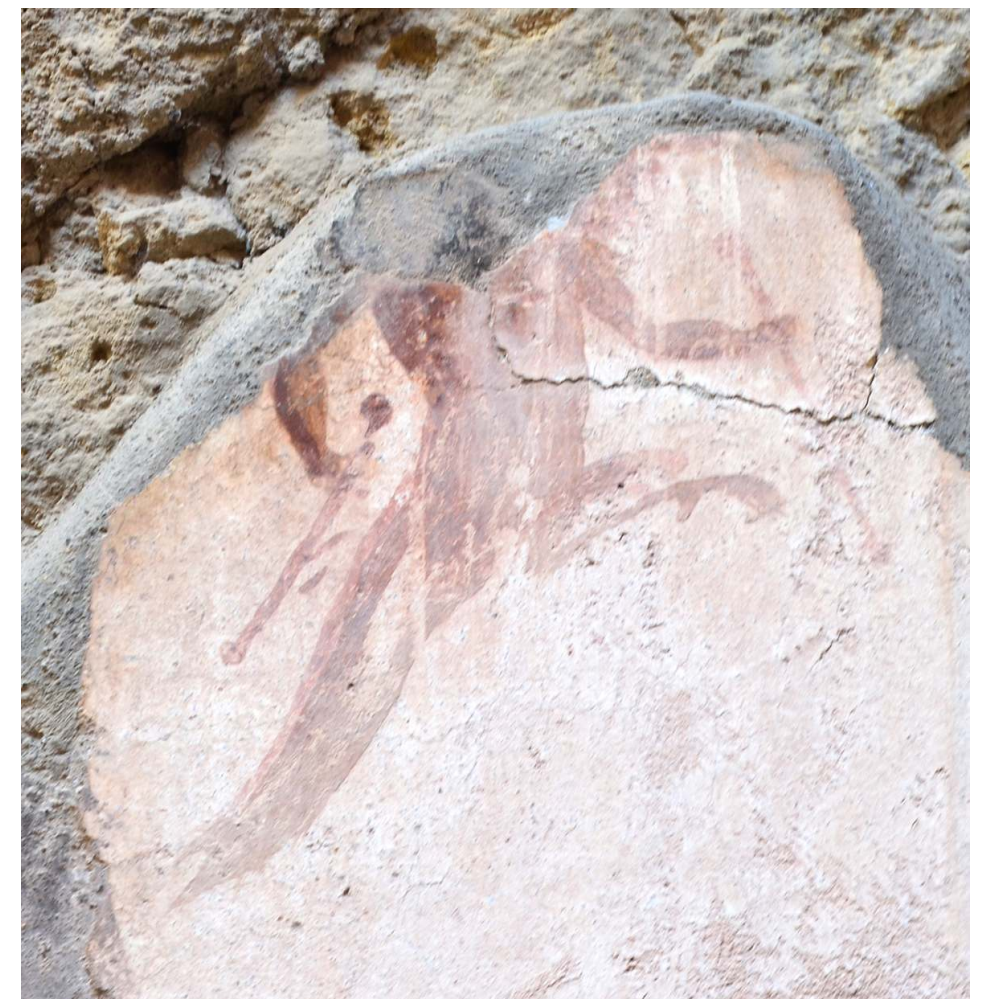

Mibact, H. Eristov, ANR Vesuvia - 2018. Licence creative commons, ( C CC BY-NC-ND.

Cette même figure ${ }^{10}$ est décrite par W. Helbig ${ }^{11}$ qui se réfère à une planche du Real Museo Borbonico accompagnée de la relation de $\mathrm{G}$. Bechi :

È un giovinetto, con gli ali omeri, uccello dalla cintura in sotto che tiene in ambe le mani una specie di flauti. Su campo bianco fu trovato dipinto in Ercolano della dimensione di poco più che cinque once di palmo. Un altro genietto mezzo fanciullo e mezzo uccello suonante una lira fu pure in Ercolano rinvenuto e si conserva fra le antiche pitture del Real Museo ${ }^{12}$.

Les caractéristiques et la hauteur $(0,21 \mathrm{~m})$ correspondent bien à celles de la figure in situ, mais la maison de Neptune et Amphitrite n'était pas dégagée en 1830-1831. Il ne peut donc s'agir que d'un élément provenant de la casa d'Argo où les fouilles avaient été reprises entre 1817 et $1855^{13}$; dans la pièce 20 , au centre de la zone supérieure du mur nord, une harpie est restée in situ, et son pendant sur le mur sud prélevé. L'étroite parenté du motif - peu courant - avec celui de la maison de Neptune et Amphitrite pourrait parler en faveur d'un même atelier sinon d'une même main.

30 À l'intérieur du IV style, l'un des types de structure de la zone médiane architecturale - le type à bipartition dans lequel une échappée centrale sépare les deux panneaux latéraux - est fréquent à Herculanum comme en témoignent la pièce 1 de la casa sannitica (V, 1.2), le nymphée de la casa dello scheletro (III, 3), l'escalier de la casa del bel cortile (V, 8), le triclinium 1 de la casa del mobilio carbonizzato $(\mathrm{V}, 5)$. 


\section{Boutique 13}

31 Cet espace $(5,20 \mathrm{~m} \times 4,10 \mathrm{~m})$, transformé en boutique dans la dernière période présente un cas intéressant en ce qui concerne tant l'histoire antique de la maison, que les choix de restauration modernes (fig. 6).

Fig. 6 - Casa di Nettuno ed Anfitrite. Pièce 13, mur est : a. état avant $62 / 63$; b. état après sa transformation en boutique ; c. reconstruction moderne suite aux fouilles d'A. Maiuri.
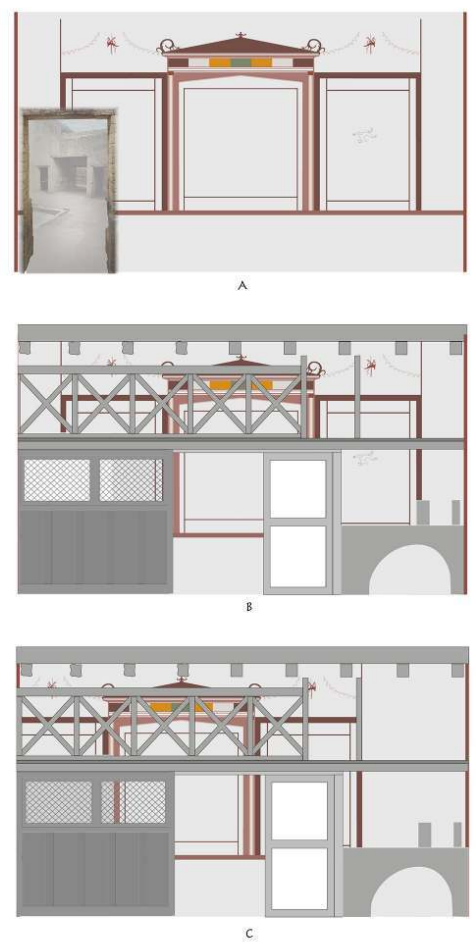

Mibact, H. Eristov, ANR Vesuvia - 2018. Licence creative commons, (c) CC BY-NC-ND.

Le mur oriental, derrière la mezzanine ${ }^{14}$ et au-dessus de son plancher, conserve les restes d'un décor préexistant, antérieur au séisme de 62/63 et à la restructuration de la pièce. Son organisation générale peut être restituée grâce à ces vestiges complétés par ceux du mur sud. La paroi à fond blanc comporte une zone basse, sans décor visible, délimitée par une bande rouge à $0,74 \mathrm{~m}$ du sol. En zone médiane, deux panneaux latéraux (larges de $1,28 \mathrm{~m}$ ) encadrés de bandes et de filets rouges et ornés d'un cygne en vol (conservé à droite du mur sud) flanquent un panneau central à couronnement en bâtière, et en forme de haut édicule large de $1,76 \mathrm{~m}$; ses deux colonnes portent un entablement divisé en caissons de couleurs alternées et un fronton à volutes en acrotères qui dépasse largement la hauteur des panneaux latéraux. En zone supérieure, un rhyton (conservé à gauche du mur est) est suspendu entre deux fines guirlandes accrochées, vers l'édicule, à la volute du fronton.

À première vue, il s'agit d'un schéma de $\mathrm{III}^{\mathrm{e}}$ style à haut édicule ${ }^{15}$; un exemple similaire, quoique plus raffiné, se trouve dans le triclinium de la casa del sacello di legno. En réalité, l'exécution rapide, la réduction des couleurs (même compte tenu de l'usure du décor), l'emploi de motifs tels que le cygne en vol sur les panneaux médians, ou le rhyton 
suspendu en zone supérieure, font pencher plutôt vers une phase de transition entre III et IV style, sans doute dans les années 40.

La restitution de ce décor fait surgir interrogations et observations. En premier lieu, dans l'état actuel de l'architecture, la limite inférieure des trous d'encastrement des poutres se situe à $2,86 \mathrm{~m}$ du sol, tandis que la hauteur maximale du décor jusqu'au faite du fronton est de $2,87 \mathrm{~m}$, non compris la hauteur d'un petit acrotère sommital dont on aperçoit l'amorce. Comme d'autre part les deux guirlandes latérales sont incomplètes, il faut que le prolongement de leur courbe jusqu'à leur point d'accroche se situe $0,10 \mathrm{~m}$ à $0,20 \mathrm{~m}$ plus haut, soit une hauteur totale restituée du décor à environ $3 \mathrm{~m}$ ou 3,10 $\mathrm{m}$. Par ailleurs, les vestiges conservés sur le mur est attestent bien la hauteur de la zone basse et celle de la bande horizontale qui couronne les panneaux médians, de sorte que l'hypothèse d'une erreur de repositionnement des plaques peintes lors de la restauration ne peut suffire à résoudre le problème. On est donc amené à supposer que lors de la restructuration de la pièce en boutique, le plancher de l'étage a pu être abaissé, ce qui offrait une meilleure hauteur pour les pièces de l'étage, ou que la grande épaisseur du plancher en béton restauré à l'étage a entraîné la disparition de la partie supérieure du décor. Malheureusement, l'état actuel de l'étude architecturale ne permet pas de trancher entre ces deux hypothèses. Seule la restitution du décor témoigne du manque de congruence entre la hauteur de celui-ci et celle de la pièce.

Par ailleurs, il y a bien eu un repositionnement moderne du décor du mur est, mais il s'est opéré dans le sens d'une translation latérale. En effet, l'édicule est, aujourd'hui, décentré vers la gauche. Une telle disposition s'expliquerait dans le cas d'une pièce nettement plus longue que large et structurée, au moins en ce qui concerne son décor, en antichambre et pièce proprement dite. Or le premier état de cet espace comportait une porte donnant sur l'atrium et ouvrant à l'angle nord ; s'il y avait eu un effet d' « antichambre », il ne pouvait donc se trouver que de ce côté, impliquant donc que l'édicule soit décentré vers le sud, et non vers le nord. Pour corriger cette aberration, il faut déplacer l'édicule et ses deux panneaux latéraux au centre de la paroi, ce qui laisse entre la limite extérieure de ceux-ci et les angles du mur une largeur résiduelle de $0,54 \mathrm{~m}$.

Selon ces hypothèses, les séquences de l'évolution du décor non seulement dans l'Antiquité, mais aussi à l'époque moderne, seraient donc les suivantes.

1) La pièce fermée sur la rue, haute de 3,00 à 3,10 m, ouvre dans l'atrium et reçoit son décor.

2) Après $62 / 63^{16}$, la pièce est isolée de l'atrium, la porte bouchée, une autre porte ouverte vers l'escalier desservant les étages (pièce 11), la façade ouverte pour transformer la pièce en boutique. Une mezzanine y est installée, elle dissimule le décor ${ }^{17}$.

3) Lors des fouilles, A. Maiuri fait restaurer la boutique et déplace les enduits pour centrer l'édicule peint en fonction de la mezzanine.

L'état actuel de la maison et de son étage sur la rue - en raison des choix de restauration qui ont été faits pour rendre visibles quelques pièces de l'étage - empêchent de valider définitivement ces hypothèses. Cependant, il n'est pas exclu qu'une reprise de l'étude architecturale de l'étage puisse apporter quelques éléments nouveaux.

\section{Façade}

En façade (L. env. 9,80 m, pan à droite de la boutique non compris), deux grands fragments ont été repositionnés de part et d'autre de la porte d'entrée; deux fragments 
plus petits se trouvent en zone haute à gauche de la porte (fig. 7). À première vue ils portent un décor plus adapté à une pièce d'habitation qu'à une façade, ce qui a suscité des interrogations : s'agit-il de fragments du décor intérieur remontés là au moment des fouilles? Ou bien d'un décor de façade totalement atypique et conçu comme un décor intérieur? L'identité de structure décorative a fait penser dans un premier temps que ces éléments appartenaient au décor des fauces ; mais il est vite apparu que ni les dimensions ni certains détails ne correspondaient et qu'il était impossible de les restituer sur le mur sud qui aurait eu une organisation différente du mur nord. Selon d'autres hypothèses, ce décor aurait pu provenir de l'étage, or les parois conservées montrent des dispositifs tout à fait différents. Aucune autre pièce de la maison n'a conservé de vestiges cohérents avec les fragments en façade, et il est exclu de supposer qu'ils proviennent d'une autre maison.

Fig. 7 - Façade de la casa di Nettuno ed Anfitrite sur le cardo IV.

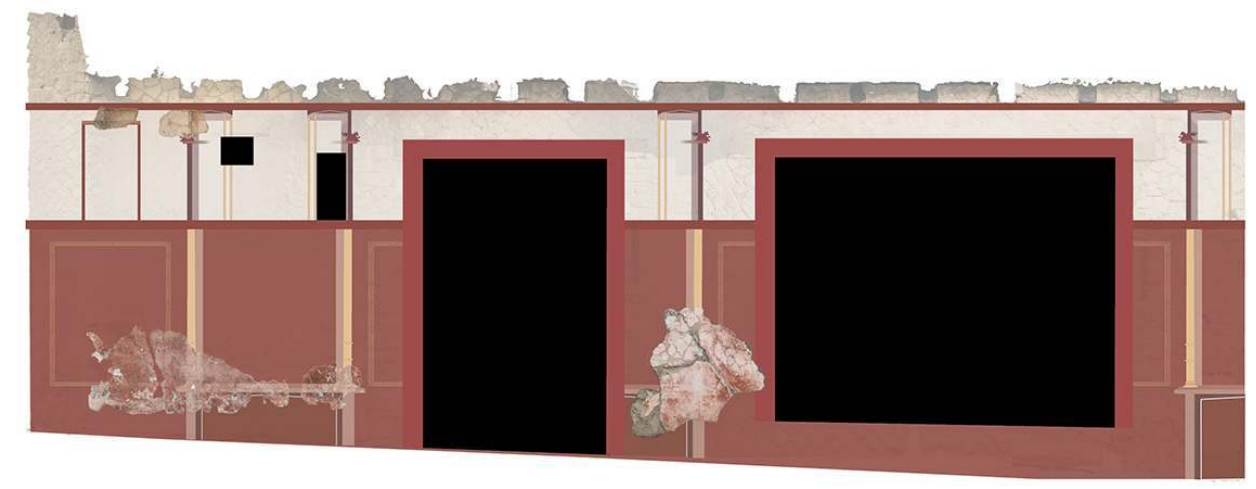

Mibact, H. Eristov, ANR Vesuvia - 2018. Licence creative commons, (c CC BY-NC-ND.

39 À gauche de la porte, un fragment à fond rouge bordeaux (h. 0,70 m, L. 2,46 m) conserve un long piédestal décoloré à corniche moulurée saillante dont la limite supérieure se situe à $0,63 \mathrm{~m} \mathrm{du} \mathrm{sol;} \mathrm{son} \mathrm{encadrement} \mathrm{noir} \mathrm{en} \mathrm{bâtière} \mathrm{enferme} \mathrm{une} \mathrm{zone} \mathrm{très} \mathrm{lacunaire.} \mathrm{Aux}$ deux extrémités de la corniche, une colonne ocre à base attique est redoublée, vers l'intérieur, par une bande verticale décolorée, l'ensemble encadrant un panneau large de 1,19 m dont l'essentiel est perdu. À gauche, la présence d'un champ médian est attestée par l'angle d'une bordure ajourée large de $4 \mathrm{~cm}$ et dessinant des pentagones irréguliers tête-bêche à petits fleurons répétitifs (type Barbet 65 a) ${ }^{18}$. Le schéma décoratif faisait donc alterner des champs encadrés de bordures ajourées, et des panneaux architecturaux dont on ne connaît que le piédestal et les colonnes. La hauteur donnée à cette zone médiane est inconnue; elle a été restituée à 1,07 $\mathrm{m}$ comme celle des fauces.

La présence d'une zone supérieure à fond blanc est attestée par deux fragments, l'un avec un griffon en acrotère tourné vers la gauche et surmonté d'un plafond à solives; l'autre, plus à gauche, avec les vestiges d'une corniche fictive et l'amorce de la bande rouge formant la limite haute du mur.

De par sa présence, le griffon suppose un entablement qui ne peut être symbolisé que par un tracé situé à 2,17 $\mathrm{m}$ du haut du piédestal; derrière lui passe le support d'un mur 
fuyant qui soutient un plafond à solives en perspective. La baie ouvrant sur cet espace n'est conservée que sous la forme d'une amorce d'encadrement en bâtière. Grâce à cette forme triangulaire, une largeur de $0,51 \mathrm{~m}$ est donnée à ce pavillon dont le support visible prolonge, semble-t-il, la colonne de la zone médiane. En revanche, l'éventuelle correspondance des autres supports (hypothétiques) avec des éléments de la zone architecturale à fond rouge ne peut être établie. Par symétrie, un autre pavillon est restitué au-dessus de la colonne médiane de droite. Il est à noter que, en façade comme ailleurs, les deux petites fenêtres coupaient partiellement le décor. Au-dessus des champs rouges médians, le fragment à fond blanc et corniche fictive peut s'interpréter comme un édicule occupant l'espace entre les paires de pavillons; si l'on connaît sa hauteur, sa largeur reste hypothétique.

Le fragment (h. 1,12 m ; L. 1,21 m) existant entre la porte des fauces et celle de la boutique, quoique très érodé, présente l'extrémité droite d'un piédestal, les traces de son encadrement noir, de la colonne et de la bande verticale qui le surmontent, ainsi que du champ rouge à bordure ajourée. Aussi est-on fondé à restituer un dispositif paratactique tout le long de la façade où se succéderaient quatre champs dont seul celui de gauche ne serait pas interrompu par une porte, et, virtuellement, trois panneaux architecturaux dont un seul complet, un autre interrompu par la porte vers les fauces, et les autres totalement escamotés par l'ouverture de la boutique.

Nous avons donc affaire à un décor de façade qui fait écho à celui des fauces, tant par son chromatisme que par la présence en partie basse de larges piédestaux portant les architectures de zone médiane ; ce que l'on peut restituer de la zone supérieure annonce également la vue de celle des fauces. Atypique dans l'état de nos connaissances sur les décors de façade, celui-ci constitue un rare témoignage de la variété qui pouvait y régner. Il révèle également le souci que pouvaient avoir certains commanditaires de préparer visuellement le visiteur et d'annoncer, dès la rue, le décor de la maison. Quelques mètres plus bas, la façade de la casa sannitica est probablement à lire dans le même sens: les restes du décor de $\mathrm{I}^{\mathrm{er}}$ style de la façade reprennent le décor des fauces avec son feint appareil de stuc en relief.

Toutefois, le décor de façade de la casa sannitica est d'un type assez usuel et pertinent à cet emplacement : les revêtements de façade en style de «grand appareil » se rencontrent fréquemment dans l'environnement urbain du monde romain -aussi bien en ornementation des bâtiments monumentaux (édifices publics, sanctuaires...) que des habitations.

Le cas du décor la façade de la casa di Nettuno ed Anfitrite, s'il est très original - puisqu'il prolonge, dans un module beaucoup plus large, le décor de IV style des fauces de la maison - n'est cependant pas un cas totalement isolé. D'autres habitations d'Herculanum présentaient un enduit de façade à décor polychrome. C'était le cas, notamment, de la casa del bicentenario, sur sa façade donnant sur le decumanus maximus. On y distingue encore la trace d'un enduit peint de carrés polychromes. Ce décor de façade est semblable à celui qui ornait, à Pompéi, la façade de la foulerie dite "de Fabius Ululitremulus " (IX 13,4), documenté par des photographies anciennes, mais aujourd'hui effacé. 


\section{Étude de l'étage - architecture et décor}

46

L'étage de la casa di Nettuno ed Anfitrite, qui compte parmi les mieux préservés d'Herculanum (fig. 1) peut être subdivisé en deux espaces séparés: d'une part les pièces 14 à 22 qui se situent au-dessus des pièces du rez-de-chaussée au sud et à l'ouest de l'atrium, ces dernières surplombant le trottoir et donnant sur la rue ${ }^{19}$, et, d'autre part, les pièces 23 et 24 aménagées au-dessus des pièces à l'est de l'atrium.

\section{Fraction occidentale de l'étage}

L'étage qui se déploie au-dessus des pièces à l'ouest et au sud de l'atrium fut largement restauré pendant les fouilles, mais de façon à ce que les pièces à l'étage soient visibles depuis la rue afin de présenter la maison au public. Plusieurs objets et pièces de mobilier découverts à d'autres endroits, dont certains sont toujours visibles, furent exposés à l'étage ${ }^{20}$. L'accès au premier étage s'effectuait par un escalier situé dans la pièce 11. Seules les trois premières marches maçonnées de cet escalier sont préservées, mais les GSE ( Giornali degli Scavi) indiquent que la partie en bois de cet escalier, détruite par le passage d'un tunnel creusé par les Bourbons, s'élevait vers l'est le long du mur sud pour aboutir dans la pièce $21^{21}$. L'étage situé au-dessus des pièces de la partie occidentale était composé de cinq pièces $(14-16,18,20)$ donnant sur la rue, incluant un couloir (19), ce dernier donnant accès aux deux pièces 21 et 22 situées respectivement au-dessus des pièces 11/5 et $6 \mathrm{du}$ rez-de-chaussée ${ }^{22}$. Les pièces de l'ensemble occidental étaient séparées par des cloisons en pan de bois hourdé, tandis que de larges piliers en opus vittatum supportaient des linteaux le long de la façade ainsi que le toit qui fut restauré23. Ce type de cloison permettait non seulement de diminuer la charge reposant sur les murs porteurs mais de plus, grâce à leur légèreté, elles ne devaient pas obligatoirement être posées au-dessus de ces derniers et permettaient ainsi un agencement des pièces qui se distinguait considérablement de celui du rez-de-chaussée ${ }^{24}$.

L'étage était soigneusement arrangé, avec un accès aux différentes pièces, facilité par le couloir prolongeant deux des pièces à chaque extrémité au-dessus de la rue (pièces 15 et 18). L'étage était dominé par une large pièce centrale (16), de toute évidence un triclinium, somptueusement décoré avec des peintures fines en IV ${ }^{e}$ style (fig. 8). Le schéma décoratif raffiné comprenait un soubassement noir ainsi que des encadrements rouges dans la zone principale, séparés les uns des autres par des échappées architecturales sur fond blanc incluant des éléments architecturaux stylisés et des centaures en acrotère. Des peintures en panneaux ornaient les champs centraux au-dessus desquels se trouvaient des aedicula et, dans la partie supérieure de la zone peinte en rouge, des pinakes blancs ${ }^{25}$. Des peintures d'une telle qualité ont été trouvées dans un quart des étages à Herculanum tandis que les pièces à manger richement décorées, comme c'était le cas ici, étaient de toute évidence souvent les plus grandes ou parmi les plus grandes et les plus importantes de la maison en termes d'interaction sociale. Il s'agissait en effet de la plus grande pièce à l'étage $(4,04 \mathrm{~m} \times 3,65 \mathrm{~m})$ et, mis à part l'atrium, de la deuxième plus grande pièce intérieure de la maison. On notera également la présence d'une petite fenêtre carrée aménagée dans le mur oriental. La quantité de lumière que celle-ci laissait pénétrer a dû être très limitée du fait qu'elle se situait sous le toit de l'atrium présent de l'autre côté du mur. En présumant qu'il y avait une fenêtre dans le mur occidental, nous pouvons supposer qu'elle

Chronique des activités archéologiques de l'École française de Rome , Les cités vésuviennes 
permettait d'éclairer la pièce 16, éventuellement à travers l'espace 17 . Il ne reste aucun indice permettant d'indiquer que la pièce était fermée, mais des vestiges ou structures observées ailleurs laissent penser que c'était le cas, témoignant de la seule utilisation d'une cloison légère ou même de rideaux afin d'en préserver l'intimité et de créer une séparation par rapport aux pièces de service adjacentes ${ }^{26}$. Aucune trouvaille n'a pu être attribuée à cette pièce, et les deux éléments de mobilier qui y sont toujours exposés ont été découverts ailleurs. Malgré l'affirmation d'Amedeo Maiuri selon laquelle ils auraient été découverts là, le pied de lit en bronze, par exemple, proviendrait plus certainement du triclinium (7) situé au rez-de-chaussée ${ }^{27}$; comme souvent à Herculanum, l'exposition d'objets dans des pièces relève plus de la mise en scène que de la recomposition à l'identique du contexte de découverte.

Fig. 8 - Casa di Nettuno ed Anfitrite. Pièce 16, mur est, peintures murales de IVe style.

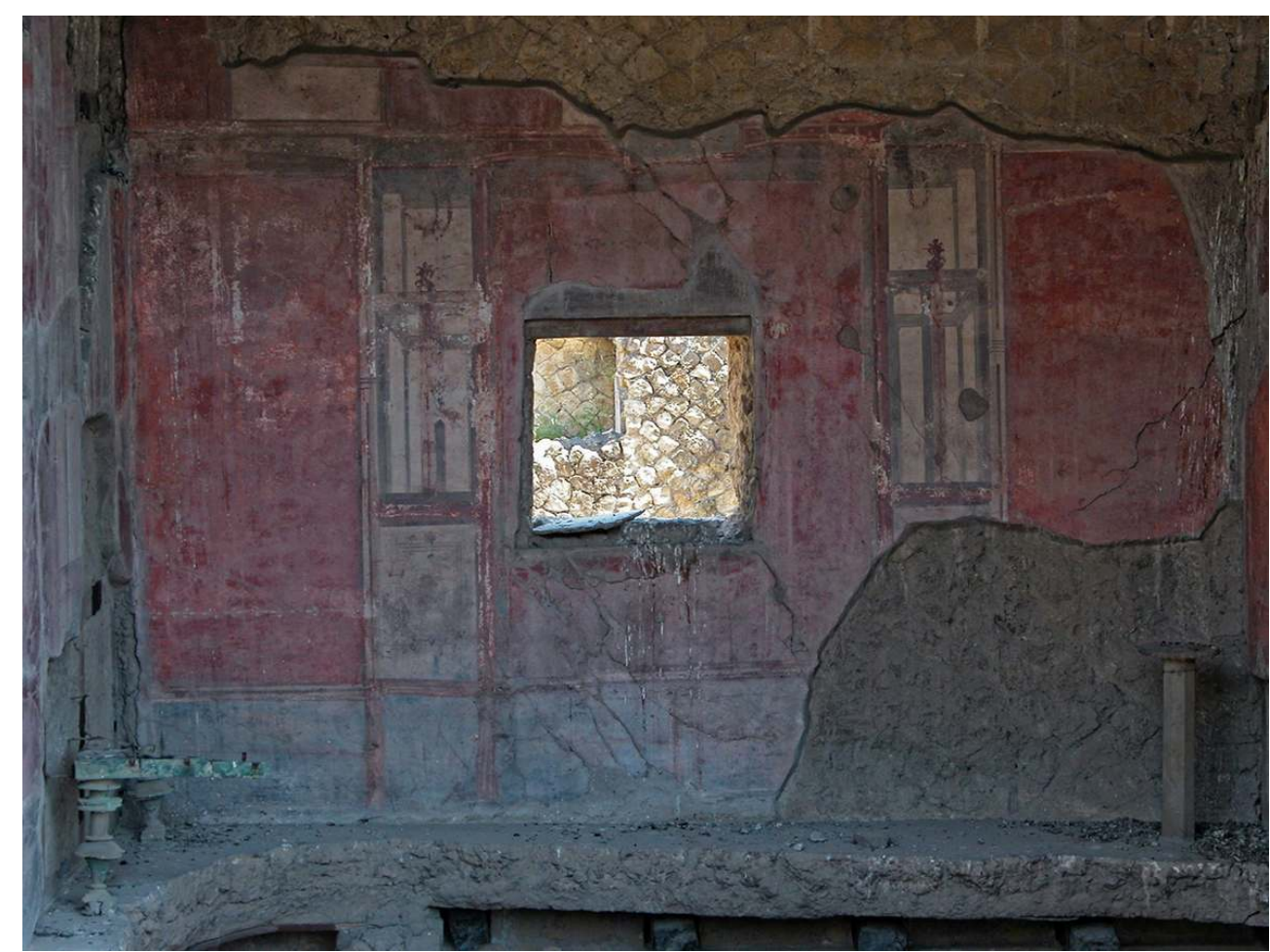

Mibact, J.N. Andrews - 2006. Licence creative commons, (c CC BY-NC-SA.

\section{Pièce 15}

Une petite pièce (15) située à l'extrémité nord de l'étage était décorée dans un $\mathrm{IV}^{\mathrm{e}}$ style plus simple, présentant notamment un soubassement noir et une zone principale rouge ne comportant aucun décor architectural, aucune fausse perspective ou panneau de peinture. La table en marbre, une pièce de mobilier associée à des objets en exposition, y fut placée peu de temps après les fouilles, mais ne fut pas découverte à cet endroit. En revanche, ce fut probablement le lieu de découverte d'une amphore en terre cuite remplie de fèves carbonisées, présentées ensuite dans les dolia de la boutique $\mathrm{V}, 6^{28}$. D'autres découvertes comprenant des vestiges d'un coffre en bois carbonisé à l'intérieur duquel se trouvaient des récipients en bronze, un candélabre, une lampe en terre cuite et à côté, des amphores contenant fèves et olives sont mentionnées dans les GSE et auraient 
été trouvées dans la partie septentrionale des espaces situés côté rue (pièces 14-15) ${ }^{29}$. Derrière la pièce 15 se trouvait une pièce plus large au plan en équerre (14), simplement peinte d'un enduit blanc, mais avec un panneau de peinture apparemment réutilisé et inséré dans le mur ouest.

\section{Pièces 18 et 20}

50 À l'extrémité sud de l'étage se situaient une cuisine étroite (20) et une latrine (18). La cuisine était équipée d'une table de cuisson maçonnée derrière laquelle se trouvait un tuyau de descente permettant probablement la conduite des eaux pluviales. À droite de cette table, sur le mur méridional, se trouve une peinture de laraire ${ }^{30}$. Si cette peinture est aujourd'hui fortement délavée, son contenu peut être reconstitué à partir de la description dans les GSE et des restes visibles ${ }^{31}$ (fig. 9). Deux serpents s'approchant d'un autel sur lequel étaient exposées des offrandes ont été perdus, bien que dans la partie conservée de la peinture, exécutée sur un fond blanc, l'on puisse distinguer un panneau avec un encadrement peint en rouge sur lequel figurent deux représentations symétriques de lares dansants ${ }^{32}$. Sous cette représentation, un foyer bas a été érigé en maçonnerie; il s'agit très probablement d'un autel dont la surface de chauffe est délimitée au moins sur trois côtés par un bourrelet en béton (fig. 10). Le reste de la décoration de cette pièce n'est que partiellement restituable. On observe encore toutefois les vestiges délavés d'un édicule à fronton se rapportant à un décor de $\mathrm{IV}^{\mathrm{e}}$ style $^{33}$, témoin d'un ancien état d'occupation de cet espace. En effet, il s'agit d'un type de décor caractéristique d'une pièce résidentielle, et non d'un espace de service comme une cuisine, pour laquelle il n'aurait jamais été spécifiquement mis en œuvre. Ce décor IV ${ }^{\mathrm{e}}$ style est donc le témoignage résiduel d'une ancienne phase d'occupation de l'étage, au cours de laquelle il n'y avait qu'une seule pièce dans la partie méridionale de l'étage donnant sur la rue. 
Fig. 9 - Casa di Nettuno ed Anfitrite. Extrémité ouest du mur méridional de la cuisine (20); vestiges d'un laraire peint.

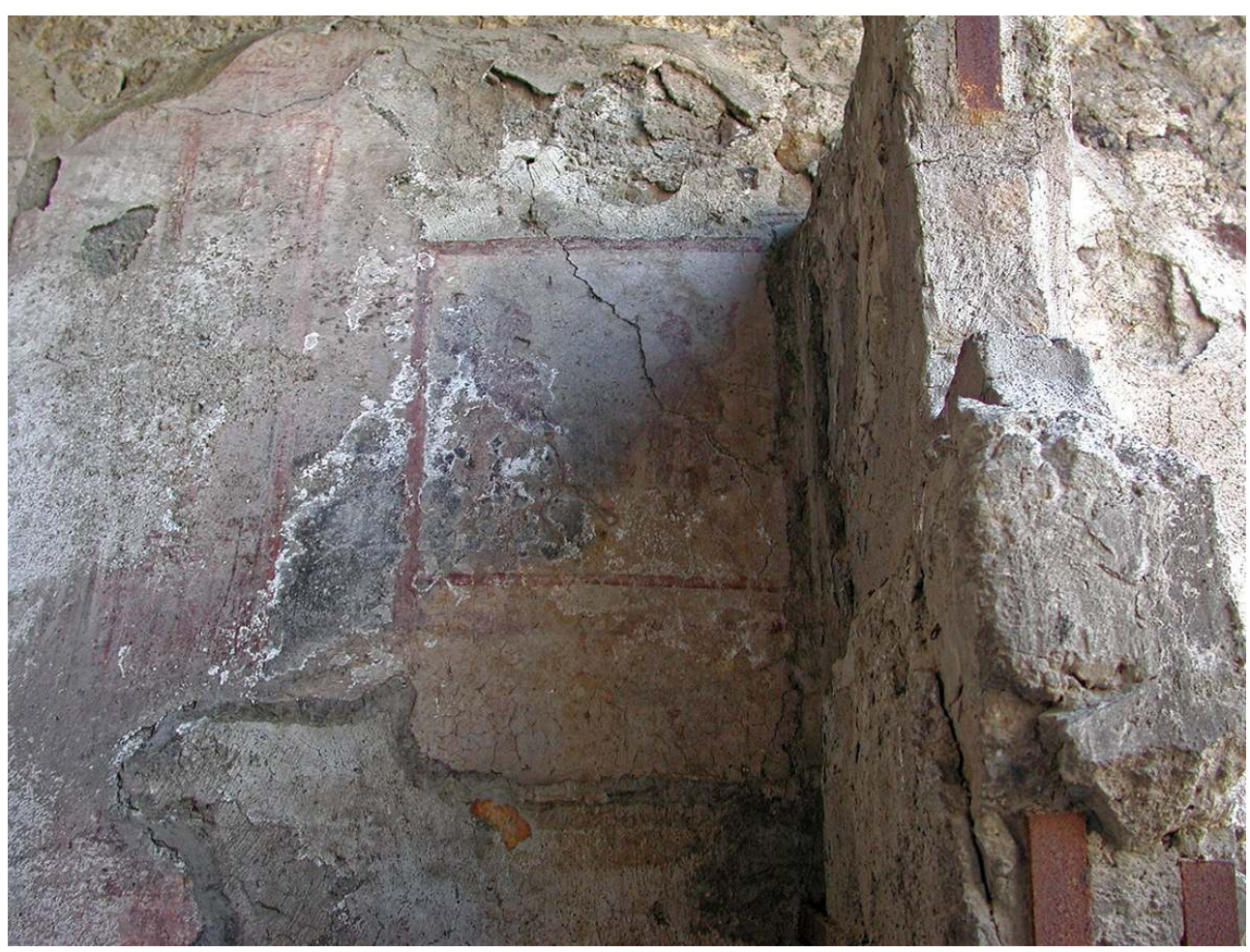

Mibact, N. Monteix - 2003. Licence creative commons, @ CC BY-NC-SA.

Fig. 10 - Casa di Nettuno ed Anfitrite. Autel maçonné sous la peinture de laraire dans la cuisine (20).

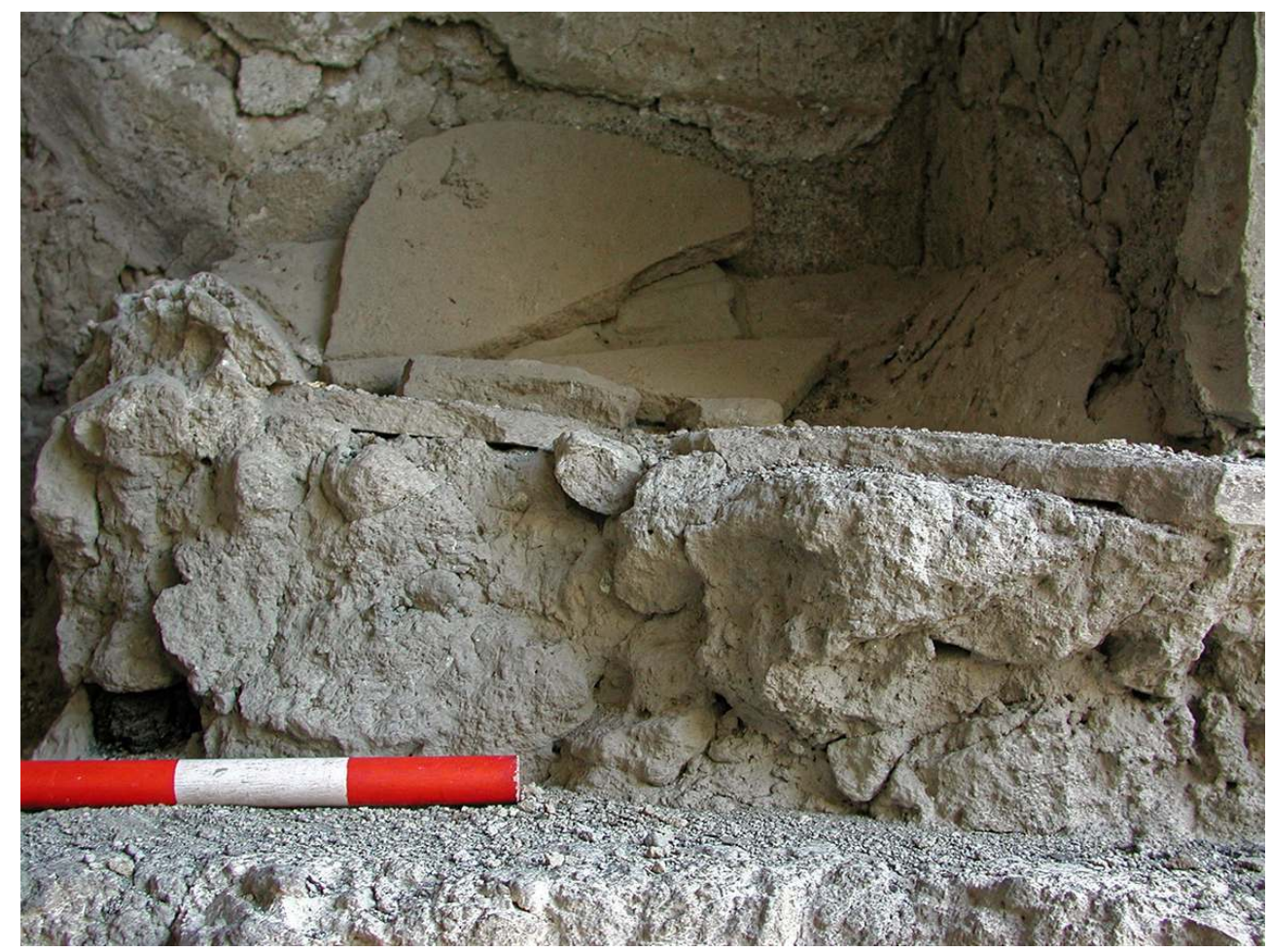

Mibact, N. Monteix - 2003. Licence creative commons, @ CC BY-NC-SA. 
51 L'édicule est, en effet, positionné au centre exact de la paroi méridionale, sans inclure la partie en encorbellement (17) ${ }^{34}$. Cette organisation de la structure du décor, mais aussi sa qualité, plaident pour que la situation originelle d'une pièce unique (19-20, voire 16-19-20) ait été recoupée dans un second temps par l'adjonction de cloison(s) en pan de bois hourdé de manière à ménager trois espaces de service : une latrine en (18), une cuisine en (20) et un couloir d'accès en (19). Par ailleurs, pour aménager la latrine, on empiéta légèrement sur le couloir en encorbellement (17) ${ }^{35}$.

Lors de cette phase de réaménagement, le décor $\mathrm{IV}^{\mathrm{e}}$ style fut conservé, au moins sur le mur méridional, contre lequel furent aménagé un banc de cuisson, un autel domestique et une latrine. L'enduit peint fut seulement partiellement modifié dans la partie occidentale de la paroi, pour y ajouter, au-dessus de l'autel domestique, une peinture de laraire.

La latrine 18 est séparée de la cuisine 20 par une cloison en pan de bois hourdé. Le siège, dont seule la partie orientale est conservée, était séparé de cette paroi par un étroit espace $^{36}$. Le siège et le dallage l'entourant sont couverts d'un béton de tuileau hydraulique. Il est possible - bien que peu probable - que la vidange de la latrine ait été alimentée par un tuyau (provenant du toit ?), dont une section isolée a été insérée entre le siège et la cloison (fig. 11). S'il se trouve dans sa position originelle, il aurait pu amener l'eau de nettoyage, ensuite évacuée et drainée par le tuyau de vidange de latrine. Cette hypothèse doit cependant être prise avec précaution : le diamètre du tuyau venant du toit (diam. interne $0,20 \mathrm{~m}$ ) est assez (trop?) important pour constituer une véritable adduction, par ailleurs vraisemblablement non pérenne.

Fig. 11 - Vestiges de la latrine située dans la pièce 18 à l'étage de la casa di Nettuno ed Anfitrite.

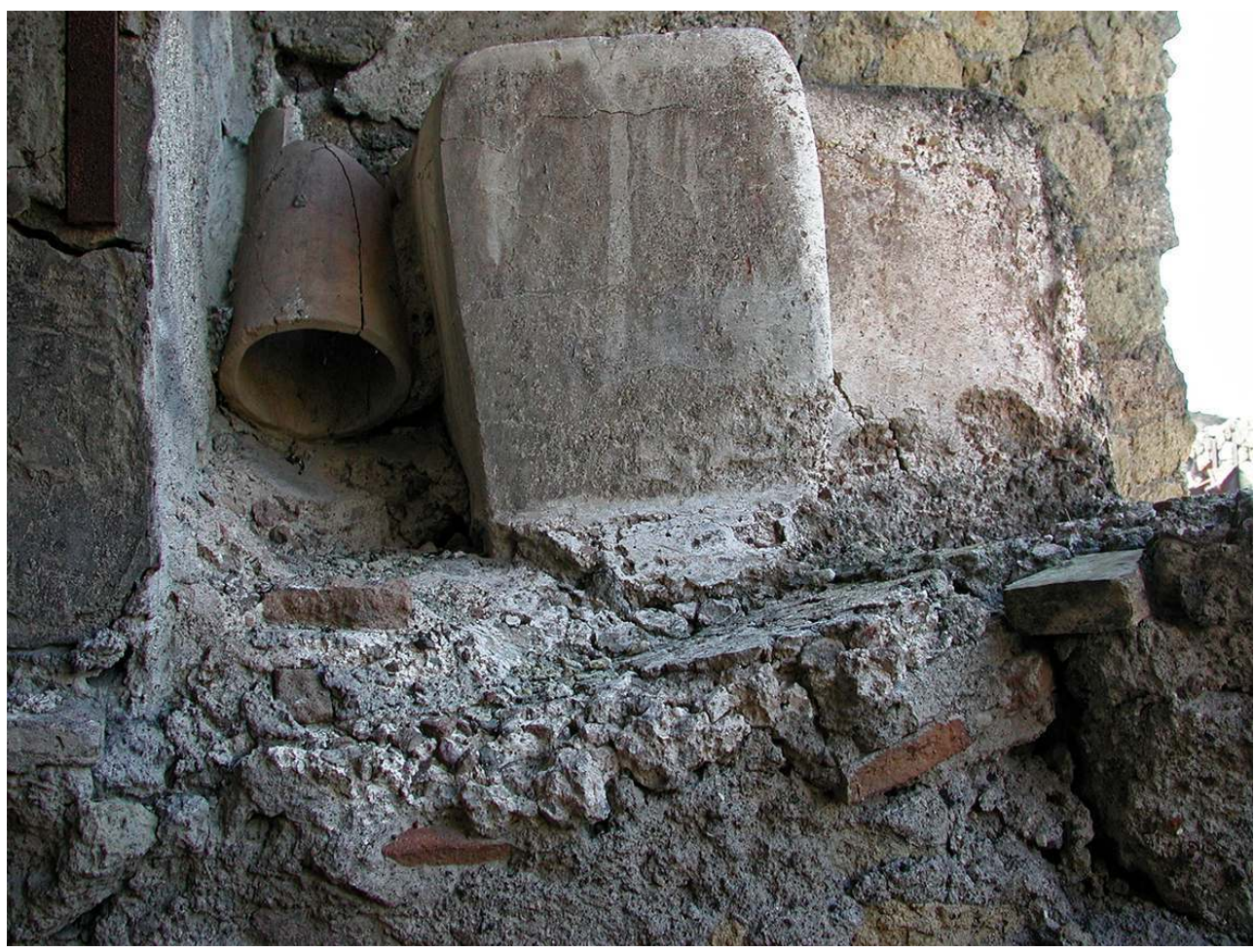

Mibact, N. Monteix - 2003. Licence creative commons, (C) CC BY-NC-SA. 


\section{Pièce 21}

\section{l'escalier, lequel devait à l'origine aboutir à quelque distance du mur ouest afin de} permettre une entrée dans la pièce. En plus de la porte qui reliait la pièce à la suite des pièces d'habitation orientée vers l'ouest, on doit supposer l'existence d'une porte supplémentaire dans le mur oriental donnant accès à la pièce 22. L'existence d'une telle porte est mentionnée dans les GSE bien qu'aucune trace n'en subsiste actuellement (annexe, description de la maison effectuée fin juin 1934). La pièce était pourvue d'un enduit simple avec un soubassement haut en opus signinum et un enduit monochrome audessus.

\section{Pièce 22}

Au-dessus de la pièce 6 , à l'étage, la pièce $22(2,33 \mathrm{~m} \times 2,50 \mathrm{~m})$ a conservé des zones significatives de son décor, même si de nombreux détails sont délavés (fig. 12). Ce sont, sur le mur est, le tiers inférieur gauche des zones basse et médiane et le tiers supérieur droit des zones médiane et supérieure ; il reste du mur sud un lambeau du haut de la zone médiane et l'angle gauche de la zone supérieure ainsi qu'un fragment du haut de la zone médiane vers le centre de la paroi. Sur le mur nord, le tiers central des zones basse et médiane est conservé, ainsi qu'un fragment de la zone supérieure sur le tiers droit. En revanche, le mur ouest est totalement perdu.

Fig. 12 - Casa di Nettuno ed Anfitrite. Pièce 22, murs nord et est, infographie de restitution du décor peint.

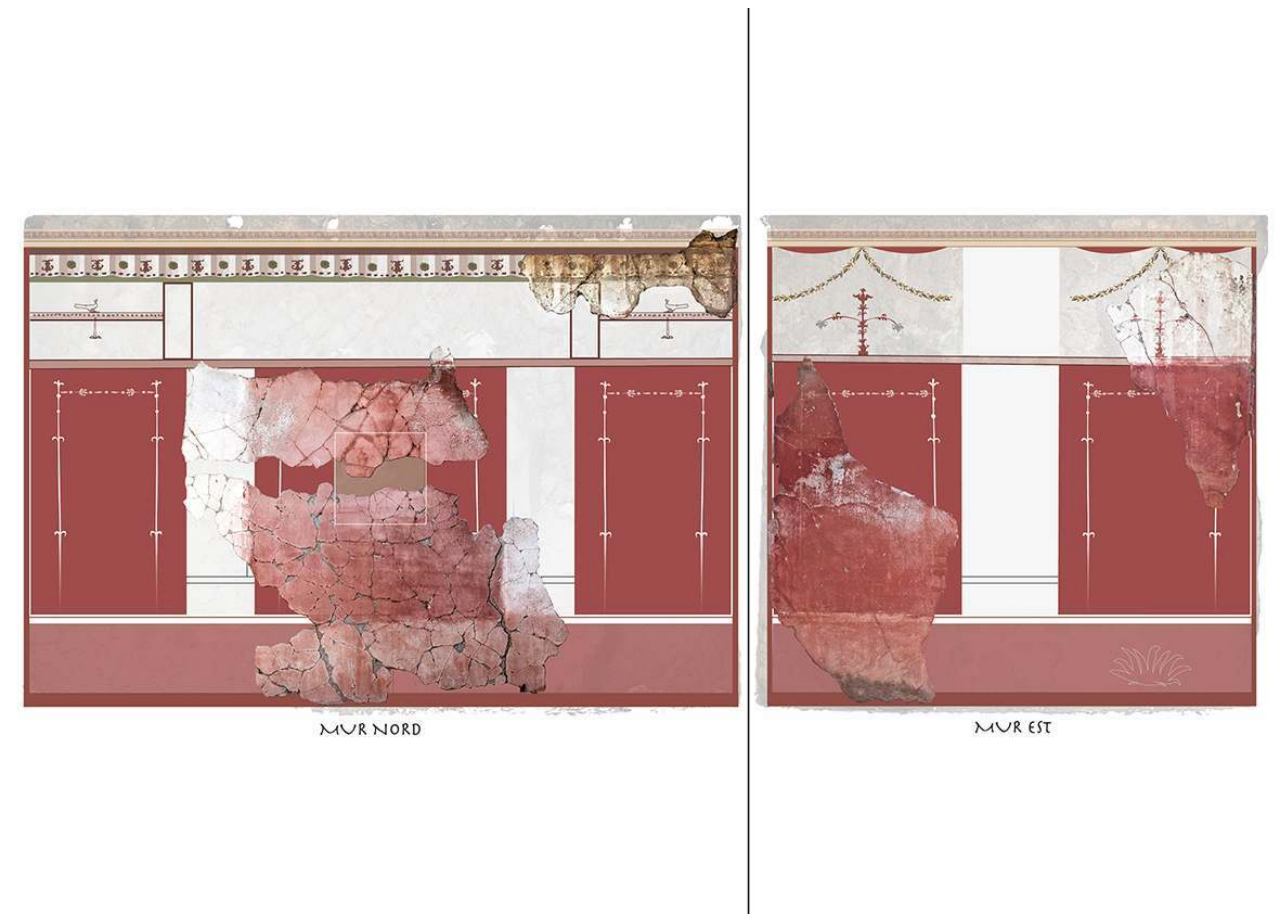

Mibact, M.-L. Maraval, H. Eristov, ANR Vesuvia - 2018. Licence creative commons, (c) CC BY-NC-ND.

Ces éléments permettent de restituer le schéma décoratif selon les principes déjà exposés pour le triclinium $7^{37}$; en effet l'identité des murs parallèles et l'organisation symétrique 
du système pariétal facilitent la compréhension d'un décor même lacunaire et valident les hypothèses. À titre d'exemple, les diverses composantes des parois nord et sud sont ici regroupées fictivement (fig. 13).

Fig. 13 - Casa di Nettuno ed Anfitrite. Pièce 22, synthèse des fragments conservés sur les murs nord et sud.

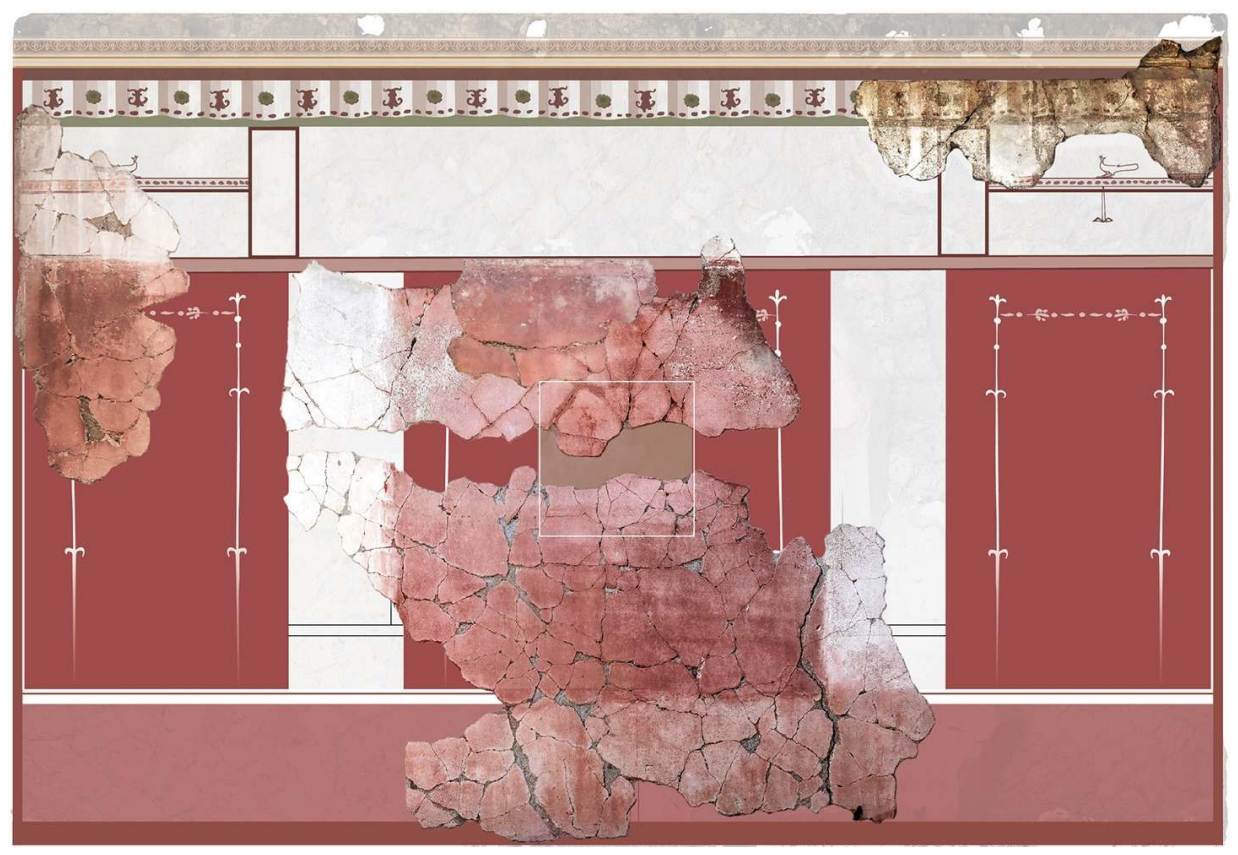

Mibact, M.-L. Maraval, H. Eristov, ANR Vesuvia - 2018. Licence creative commons, @ CC BY-NC-ND.

Le soubassement décrit comme rouge et blanc au moment de la fouille, désormais seulement rouge (h. $0,47 \mathrm{~m}$ ), comporte des touffes de feuillages, dont l'une est encore lisible sur le mur est; les articulations verticales qui devaient exister entre des panneaux sont aujourd'hui illisibles à l'exception de trois filets verticaux au centre de la paroi nord. Une fausse moulure sépare ce soubassement de la zone médiane (h. $1,30 \mathrm{~m})$ où alternent des panneaux blancs et rouges; sur les murs est et ouest, un panneau blanc central $(1.0,50 \mathrm{~m})$ sépare deux larges panneaux rouges, tandis que sur les murs nord et sud, trois panneaux rouges sont séparés par deux étroits panneaux blancs (l. environ $0,35 \mathrm{~m}$ ) dont toute trace de décor a disparu mais qu'il faut interpréter comme des échappées ${ }^{38}$. Les panneaux rouges sont animés par des tiges verticales, visibles sur le mur est, dont la base n'est pas conservée et que relie, en haut, une guirlande tendue encore à peine discernable.

$\mathrm{Au}$ centre du mur nord, se distingue, sur le panneau rouge, la ligne horizontale supérieure d'un tableau restituable sur environ $0,47 \mathrm{~m}$ de côté; au-dessous, une forme pointue correspond à un vase que les GSE mentionnent comme inséré dans un paysage sacro-idyllique ${ }^{39}$.

59 La zone supérieure à fond blanc $(\mathrm{h} .0,60 \mathrm{~m})$ couronnée par une moulure de stuc offre encore des vestiges de son décor. Sur les murs est et ouest, les tiers latéraux comportent un candélabre bas d'où partent deux tiges souples; au-dessus, une fine guirlande à double feston est accrochée à un motif rouge incurvé évoquant schématiquement un rideau. Le 
centre de la zone est perdu. Sur les murs nord et sud, l'amorce d'un étroit compartiment rectangulaire large de 0,14 $\mathrm{m}$, à $0,71 \mathrm{~m}$ de l'angle droit de la paroi structure le tiers de la zone supérieure: à mi-hauteur court une bordure ajourée horizontale à gouttes sur laquelle marchent des oiseaux ; la queue d'un paon a pu être repérée sur le mur sud, ainsi que les restes d'un possible nœud à coques et de rubans. Un petit objet devait donc se trouver suspendu sous le paon.

Sur toute la longueur des murs nord et sud court un motif de rideau blanc; le traitement en quatre bandes de valeur dégradée donne un effet ondulé accentué par la série de gouttes rouges qui suivent les courbes du bord inférieur du rideau; dans ces plis réguliers alternent bucranes rouges et rosettes vertes. Une large bande rouge puis une bande blanche cernée de filets jaunes séparent ce rideau de la moulure de stuc à palmettes circonscrites séparées par des trifols, dont un vestige subsiste à l'extrémité droite du mur nord; le parti pris de restitution consiste à répéter le même motif de palmettes, dans l'ignorance d'une possible alternance.

61 La restitution du mur ouest reprend le système du mur est, en supposant une largeur de porte identique à celle de la pièce 6 au rez-de-chaussée ; la porte ouvrait directement dans l'angle.

Des irrégularités de dimensions sont à signaler : dans l'état actuel des restaurations faites peu après les fouilles, le panneau rouge central du mur nord, large de 1,30 m, est décalé vers la droite de $0,15 \mathrm{~m}$; or un petit tracé vertical probablement préparatoire se distingue en bas de la zone médiane et correspond au centre du mur mais non au centre du panneau. On admet donc qu'il faut repositionner tout le bloc du soubassement et de la zone médiane. La largeur minimale des échappées étant connue $(0,33 \mathrm{~m})$, elles sont restituées à environ $0,35 \mathrm{~m}$, et l'alignement de l'étroit compartiment de la zone supérieure avec le bord extérieur des échappées médianes confirme ces hypothèses. La largeur des champs latéraux, bande d'angle non comprise, est de $0,77 \mathrm{~m}(0,81 \mathrm{~m}$ au total), ce qui permet d'y installer les mêmes hampes végétales que sur le panneau central.

Une autre irrégularité, due à une négligence des décorateurs, touche à la hauteur des bandes et rideaux de la zone supérieure : sur le mur sud, ils sont $3 \mathrm{~cm}$ plus haut que sur le mur nord. Sous le plafond moderne reconstruit à $2,62 \mathrm{~m}$, la hauteur restituée du décor est de $2,50 \mathrm{~m}$.

L'homogénéité décorative déjà signalée pour les pièces de la casa di Nettuno ed Anfitrite se vérifie encore une fois : la structure à bipartition adoptée dans la pièce 6 se retrouve ici. Aucun pendant n'a été retrouvé au MANN pour le paon et la bordure à gouttes. Quant au motif du rideau, très apprécié à Herculanum, il est traité ici d'une façon voisine de ce que l'on observe sur la zone basse de la diaeta de la casa del gran portale, au-dessus du compartiment à masque ; en zone supérieure de la pièce 19 de la casa dell'alcova, ce même rideau comporte aussi une bordure à gouttes.

Par ailleurs, signalons que les restes d'enduit sur le mur nord confirment que la pièce ne comportait aucune fenêtre donnant sur l'atrium et devait être éclairée artificiellement. Enfin, concernant l'instrumentum et le mobilier qui pouvaient s'y trouver, il est difficile d'attribuer des trouvailles aussi bien à cette pièce qu'à celle située à l'ouest (21), bien qu'un fragment de pied de lit en bronze et d'autres objets aient probablement été trouvés à cet endroit ${ }^{40}$. 


\section{Fraction orientale de l'étage}

Amedeo Maiuri ne donne aucun renseignement sur l'étage situé dans la partie arrière de la maison (fig. 1). Cet espace était composé de deux pièces (23-24) moins bien conservées que celles de la partie occidentale: la pièce 23 , située au-dessus du tablinum 3 et du couloir 9 , et la pièce 24 , située au nord, au-dessus de la pièce 4 . Ces deux espaces étaient reliés par une porte ménagée au centre du mur de refend. Un dallage moderne a été reconstruit pour donner un "plafond» aux pièces situées au rez-de-chaussée, à une hauteur approximative de 3,50 $\mathrm{m}$ au-dessus de celles-ci. La question de l'accès à cette partie de l'étage est problématique, étant donnée l'absence de trace indiquant la présence d'une cage d'escalier et considérant que cette partie de l'étage ne pouvait pas être reliée aux pièces 21 et 22 au sud de l'atrium à cause de l'extension en hauteur du triclinium 7 qui rendait impossible toute communication avec la partie occidentale de l'étage. Cependant, le côté est de chacune de ces pièces était initialement pourvu d'une large ouverture permettant un accès depuis la petite cour $8^{41}$. L'ouverture dans la pièce 24 fut condamnée lors de la phase finale, tandis que celle présente dans le mur est de la pièce 23 a dû rester ouverte, et l'accès à cette pièce se faisait probablement par une échelle amovible placée devant la fenêtre du tablinum. C'est en tous cas l'hypothèse que les fouilleurs avancent dans les $G S E^{42}$. Des restes d'enduit sont conservés dans les deux pièces et permettent de déterminer que la pièce 23 était décorée d'un simple enduit blanc, avec une bande rouge séparant le soubassement de la zone médiane (fig. 14), tandis que, dans la pièce 24, un soubassement peint en rouge était séparé de la zone médiane monochrome blanche par une bande horizontale. Trois groupes de graffiti furent découverts sur le montant nord de l'ouverture dans la pièce $23^{43}$. Le premier de ces graffiti mentionne une date à laquelle un esclave a reçu du vin de la part de son maittre ${ }^{44}$. Le deuxième, situé immédiatement sous le premier, correspond à un inventaire de mobilier qui a été noté sur deux colonnes, la première composée de seize lignes et la seconde de trois lignes..$^{45}$ Le troisième graffito, hapax parmi les graffiti d'Herculanum et de Pompéi, organisé en six colonnes - dont les quatre premières sont encerclées par un trait continu - de quatre lignes, correspondrait plutôt à un exercice de grammaire ${ }^{46}$. 
Fig. 14 - Casa di Nettuno ed Anfitrite. Pièce 23, vue en direction du nord est ; on distingue, à droite, le passage vers la pièce 24 et la position de l'ouverture originelle vers la cour, par une échelle.

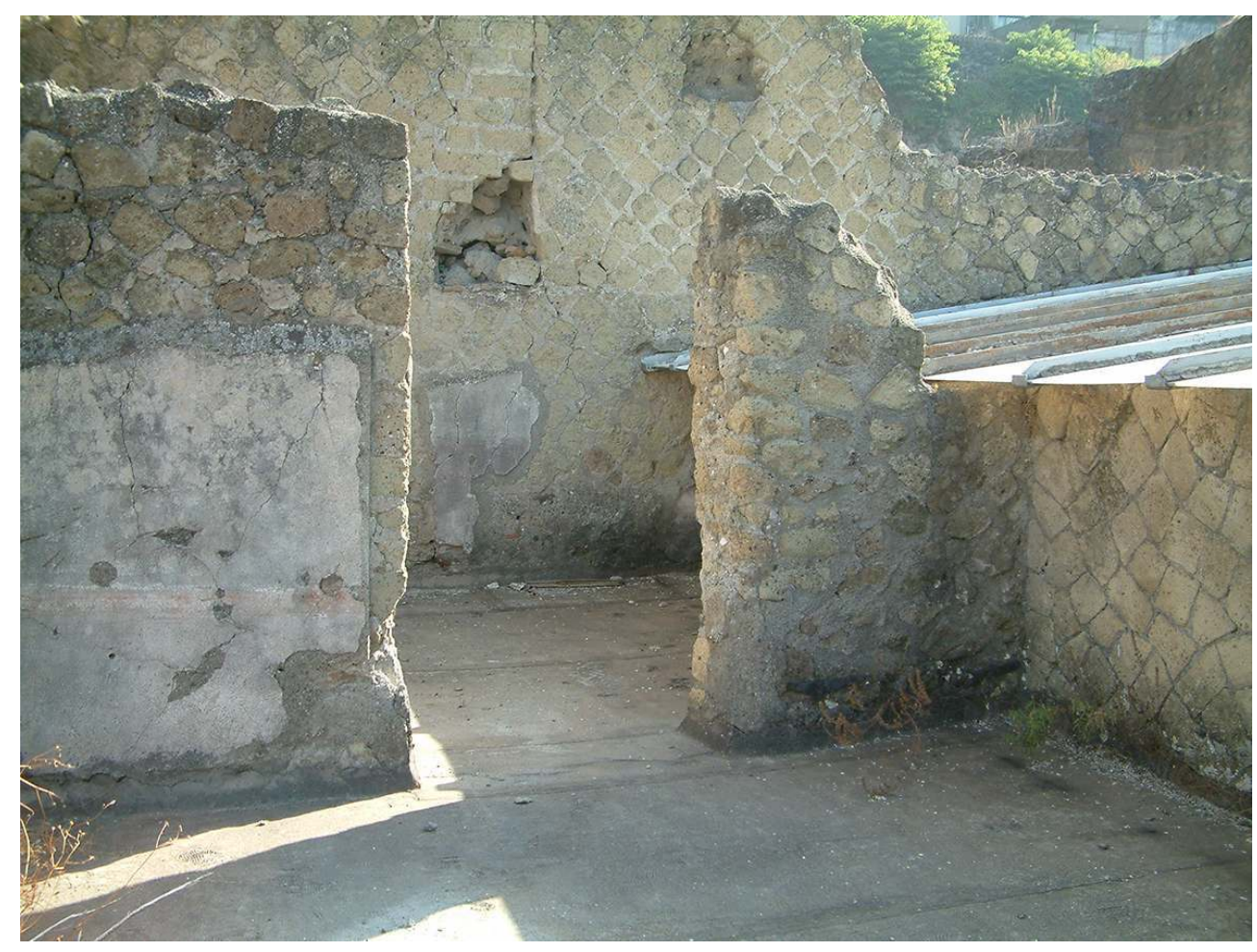

Mibact, J.N. Andrews - 2002. Licence creative commons, (c) CC BY-NC-SA.

67 Le premier texte, par la mention du dominus, renvoie immédiatement à la présence d'esclave(s) dont un(e) a incisé le texte. L'inventaire, loin de pouvoir constituer une liste exhaustive des différents objets de la maison, pointe vers des activités relativement précises: la gestion de l'eau, de son prélèvement (hama, hamula) à son stockage et son service (aquarium, urceolus, pelvis, guttus, scaphiolum, aeneum) ${ }^{47}$ et l'éclairage (candelabrum, lucubratorium, lucerna). Il pourrait s'agir d'objets utilisés dans le cadre d'activités serviles ${ }^{48}$ , ayant été confiés à l'auteur de ce texte, vraisemblablement par son maître. Quant au troisième texte, il renverrait à un exercice plutôt mnémotechnique, éventuellement lié à un perfectionnement de la langue, par ailleurs plutôt bien maîtrisée dans les autres textes. D'une manière générale, ces inscriptions tendraient à aider à caractériser ces deux pièces particulièrement difficiles d'accès : le lieu de résidence du personnel servile de la maisonnée.

\section{Remarques conclusives sur l'étage}

68 L'étage de la casa di Nettuno ed Anfitrite a fourni des indications précieuses quant à l'usage potentiel de ces espaces dans la maison romaine. Les deux logements présents à l'étage ainsi que la mezzanine dans la taberna totalisaient une surface d'utilisation d'environ $100 \mathrm{~m}^{2}$ et avec un rez-de-chaussée de presque $200 \mathrm{~m}^{2}$, ils constituent un tiers de la surface d'habitation totale de la maison. De toute évidence, le propriétaire de la maison avait besoin d'un grand étage afin de compenser le manque de place disponible au rez-dechaussée, au point de s'étendre au-dessus de la rue face aux contraintes architectoniques interdisant un étage unitaire. Conjointement à cela, les types de pièces présentes 
suggèrent une utilisation dynamique des espaces dans l'ensemble de la maison. À l'exception de l'atrium et de la cour, la plupart des différentes pièces au rez-de-chaussée possèdent leur pendant à l'étage et permettaient ainsi de dupliquer les activités qui se déroulaient au rez-de-chaussée. Il était même possible de faire de la cuisine et de manger à l'étage indépendamment du rez-de-chaussée, ce qui est une caractéristique observée dans d'autres étages à Herculanum. Cela soulève la question de l'usage de l'étage : servaitil à accueillir une maisonnée plus grande que supposé initialement? Andrew WallaceHadrill a suggéré le terme de "maisonnée élargie ", à composition variable. En plus de la famille, celle-ci était constituée d'esclaves, d'affranchis, de travailleurs, d'amis et/ou de locataires ${ }^{49}$, en particulier dans les maisons les plus importantes en termes de superficie. Alternativement, on peut aussi présumer un usage temporel de l'étage qui pouvait changer en fonction des occasions, de l'heure, de la journée ou de la saison ${ }^{50}$. En hiver, les occupants de la suite de pièces fermées donnant sur la façade pouvaient passer de pièce en pièce sans avoir à traverser un espace à l'air libre - non chauffé, au contraire du rezde-chaussée où tous les déplacements passaient obligatoirement par l'atrium. Le logement à l'étage dans la partie arrière de la maison semble n'avoir été accessible que par une échelle, ce qui laisse supposer une fréquentation moins importante, vraisemblablement plutôt dévolue au personnel servile.

\section{Annexes - Giornali di scavo di Ercolano (GSE) concernant les étages}

\section{Description finale}

69 Casa n. 6 e 7 posta sul quarto cardine lato est Insula centrale ${ }^{51}$. Incominciato lo sterro il 15 novembre 1932, terminato lo sterro il 12 maggio 1934. La casa è composta di 13 ambienti terranei e 9 ambienti superiori. Questi ultimi, 5 affacciano sulla strada, quarto cardine, e gli altri nella parte interna. Quelli esterni non hanno comunicazione tra loro, ma a ciò provvede un grande corpo avanzato sostenuto dal prolungamento dei travi interni. Vi sono quattro cucine, tre al piano terraneo e una al piano superiore. [...] La casa doveva appartenere ad un negoziante sia per un termopolio che è l'unico ambiente che tiene il vano nella strada e sia per molti graffiti trovati sul pilastro nord dell'ambiente che si sviluppa sul tablino. [...]

Piani superiori. Gli unici discretamente conservati sono quelli esterni: due stanze, un corridoio ed una cucina. Il corridoio tiene al lato sinistro la cucina e finisce sul balcone. Gli ambienti non sono in comunicazione tra loro e vi si accede mediante il balcone. Le pareti sud e nord sono a graticcio. Nella cucina accanto al focolaio vi è il dipinto comune del serpente agatodemone con i due lari. La decorazione dell'ambiente centrale è a fondo rosso con piccoli quadretti con natura morta, mentre gli altri sono con intonaco grezzo. Come già ho detto vi si saliva mediante la scala interna posta nell'ambiente n. 2 .

\section{Accès à l'étage}

\section{Pièce 11}

8 maggio 1933. Anche l'ambiente $\mathrm{N}^{\circ} 2$, della casa n. 6 sul IV Cardine, lato Est, è stato svuotato. In esso vi è la scala che mena al II piano, lato esterno ed una seconda cucina. Tiene due vani, uno sulla parete Ovest ed è comune con l'ambiente $\mathrm{N}^{\circ} 1$ e l'altro sulla 
parete Nord che mena nell'ambiente $\mathrm{N}^{\circ}$ 3. Il II vano è alto m. 1.85 e largo m. 0.92. L'Ambiente misura m. 2.50 di larghezza per m. 2.26 di lunghezza. I primi due scalini sono di muratura e ciascuno misura m. 0.66 di lunghezza, m. 0.30 di larghezza e m. 0.16 di altezza. Il ballatoio è largo m. 0.82 , alto m. 0.52 e lungo m. 1.15. Da questo punto sviluppava la scala di legno, ma nessuna misura può segnarsi perché completamente distrutta da cunicoli [...].

[Description finale (insérée après juin 1933)] Ambiente n. 2. [...] L’ambiente serviva per la cucina e la scala che mena sia ai piani superiori esterni che interni lato sud. I primi due scalini sono di muratura ciascuno misura m. 0.66 di lunghezza, m. 0.30 di larghezza e m. 0.16 di altezza. Da questo punto si sviluppava la scala di legno, ma nessuna traccia può segnarsi perché completamente distrutta da cunicoli.

\section{Pièces indéterminées}

7129 aprile 1933. Sul piano superiore della casa $6,4^{\circ}$ cardine, lato est è stata sterrata: [Description des objets inventoriés 1078 à 1080].52

1078 [= 76355] (29/04/1933): Terracotta. Lucerna monolichne col rostro accennato. Misura m. 0.125 di lunghezza e m. 0.06 di diametro. Sul fondo superiore si osservano gli attributi di Giove.

1079 [= 76356] (29/04/1933) : Terracotta aretina. Coppetta con qualche pezzo mancante. $\grave{E}$ alta m. 0.05 e del diametro di m. 0.10 .

1080 [= 76375] (29/04/1933): Terracotta. Pelvis lunga m. 0.35 e profonda m. 0.07 e del diametro di m. 0.21. Tiene il seguente bollo: VIT.

\section{Façade occidentale}

15 novembre 1932. Continua lo sterro del piano superiore sia della casa n. 5 che di quella numero 6. Ambedue case hanno il secondo piano fatto a graticcio con vari ambienti di uso incerto. Essi sono a forma rettangolare di misure varie. Nessun trovamento.

16 novembre 1932. È stato sospeso lo sterro del IV cardine lato nord che, ed è stato intensificato nelle case numero 5 e 6 piano superiore, al lato est dello stesso cardine. Nessun trovamento.

17 novembre 1932. Nessun trovamento nello sterro che si esegue nei piani superiori nelle case numero 5 e 6 , poste sul IV cardine lato est.

18 novembre 1932. [...] Sul piano superiore della casa $n .6$ nessun trovamento.

30 novembre 1932. [...] Riassunto dei lavori di sterro fatto durante il mese di novembre 1932. [...] Si è incominciato lo sterro sia del piano superiore che inferiore della casa n. 6 sullo stesso cardine ad est. Parecchi oggetti di bronzo e qualche commestibile a suo tempo furono raccolti sopra la terrazza o balcone della suddetta casa.

9 gennaio 1933. Si lavora sul piano superiore, lato esterno della casa $\mathrm{N}^{\circ} 6$, sul IV cardine lato est. È stato raccolto [Description d'un fragment de tuile marquée].

14 gennaio 1933. Sul piano superiore della casa $\mathrm{N}^{\circ} 6$, posta sul IV cardine lato est si è raccolto: [Description d'un fragment de tuile marquée].

\section{Objets découverts dans des pièces indéterminées de l'étage}

73 Sans inventaire (09/01/1933) : Un frammento di tegola di terracotta col seguente bollo: IGILLIIIIIIO53. 
Sans inventaire (14/01/1933) : Una parte di anfora di terracotta con le seguenti di lettere fatte con pittura rossa: OVIT' ${ }^{54}$.

\section{Autres}

19 giugno 1933. Nell'ambiente $\mathrm{N}^{\circ} 16$, della casa n. 6, sul IV Cardine, lato Est, a m. 1.25 dalla parete Sud a m. 0.80 da quella Est ed a m. 0.37 di altezza dal suolo del pavimento è stato raccolto: [Description de l'objet inventorié 1098] ${ }^{55}$.

1098 [= 76375] (19/06/1933) : [...] Bronzo - Leone lungo m. 0.07 ed alto m. 0.032. La coda la tiene attorcigliata e l'estremità poggia sul dorso. L'animale è in atto di slancio. Il muso è attraversato da due buchi, ciò dimostra che doveva starci qualche anello.

\section{Pièce 16}

Ambiente n. 4. [...] L'altezza dell'ambiente è di m. 2.83 ed il solarino del piano superiore era sostenuto da tre travi a forma rettangolare disposti da est ad ovest.

\section{Pièces 14-15}

7 novembre 1932. Sterrando il IV cardine lato nord, abbiamo posto alla luce un balcone che fa parte di una casa non numerata perché il vano d'ingresso è ancora otturato dal materiale. Aderente alla parete nord del balcone ed a m. 0.80 dalla parete est vi era una cassa di legno carbonizzato. Misurava m. 0.30 di larghezza, 0.98 di lunghezza e m. 0.45 di altezza, non è stato possibile conservarla perché completamente rotta. Nell'interno vi erano i seguenti oggetti: [Description des objets inventoriés 932 à 938]. Una discreta quantità di anfore di terracotta, completamente rotte stavano accatastate tra la parete nord ed est. Una di esse conteneva: [Description de l'objet inventorié 939]. In un'altra: [Description de l'objet inventorié 940] ${ }^{56}$.

1 maggio 1933. Si lavora sul IV Cardine lato Nord. Sulla terrazza esterna della casa n. 6, estremità Nord, è stata raccolta: [Description d'une amphore avec des inscriptions peintes $]^{57}$.

[Description finale (insérée après juin 1933)] Ambiente n. 1 [situé en dessous des pièces 14-15 (...)] L'ambiente è alto m. 2.89 e il solarino è sostenuto da otto travi.

\section{Objets découverts dans les pièces 14-15}

932 [= 76209] (07/11/1932) : Bronzo. Patina con piede basso. Misura m. 0.275 di diametro e m. 0.085 di profondità. Il piede è alto m. 0.02 e del diametro di m. 0.08 .

933 [= 76210] (07/11/1932) : Bronzo. Lagena monoansata alta m. 0.25 e del diametro della bocca di m. 0.07 .

934 [= 76211] (07/11/1932): Bronzo. Lagena monoansata col fondo rotto e distaccato. Misura m. 0.22 di altezza per m. 0.06 di diametro.

935 [= 76212] (07/11/1932): Bronzo. Vaso imitazione Guttus, alto m. 0.18 e del diametro della bocca di m. 0.07 .

936 [= 76213] (07/11/1932): Bronzo. Cassarola con ansa allungata contenente nell'interno un altro oggetto di bronzo che non è stato possibile distaccarlo per la forte incrostazione ${ }^{58}$ - Misura m.0.23 di lunghezza ansa compresa, m.0.06 di profondità, e m. 0.115 di diametro. 
937 [= 76214] (07/11/1932): Bronzo. Candelabro con piattello e parte del piede. Il fusto è fatto a canna smontabile e si divide in quattro pezzi collegati tra loro a mezzo di vite che trovasi all'estremità inferiore di ciascun pezzo. L'altezza è di m. 1.30. Il piattello superiore misura m. 0.10 diametro.

938 [= 76215] (07/11/1932): Terracotta. Lucerna a forma quadrata, col rostro prolungato e mancante dell'ansa. Inventario n. 938. Misura m. 0.045 per lato, ed è lunga, rostro compreso, m. 0.085. Nel fondo inferiore vi è il seguente graffito: [Retranscription d'un graffite fait avant cuisson $]^{59}$ Misura m. 0.03 di lunghezza e m. 0.005 di altezza.

939 [= 76216] (07/11/1932): Commestibili. Fave kg $13^{60}$.

940 [= 76217] (07/11/1932): Frutta. Olive chilogrammi $0.500^{61}$.

Sans inventaire (01/05/1933) : [...] Un'anfora contenente fave carbonizzate. Nella parte anteriore tiene le seguenti lettere fatte con pittura nera. In alto: [transcription fautive de CIL IV, 10777]. Lunga m. 0.18 ed alta m. 0.09 ... e più basso: XXXIV. Lunga m. 0.095 ed alte m. $0.02^{62}$.

\section{Pièce 20}

7814 novembre 1932. [...] Il piano superiore di una casa, non ancora sterrato il vano d'ingresso, e perciò non numerato, ma certamente sarà il numero 6 , è fatto tutto di opera graticcia. Difatti a m. 1.50 dalla parete sud, sulla quale vi è dipinto il serpente agatodemone in atto di accostarsi ad un'aretta colma di natura morta per mangiarla e più lontano un altro quadretto con dipinto di due lari con il corno e il piattello, si sviluppa una parete ad opera graticcia dello spessore di m. 0.12. Ogni blocco misura m. 0.56 per 0.56 ed il legno di sostegno è di m. 0.10 per 10 quello verticale, e di m. 0.08 per 0.10 quello orizzontale. L'altezza attuale è fatta di tre blocchi che complessivamente ammontano a m. 1.98 di altezza. All'estremità ovest vi è un sol filare di blocchi e poscia vi è un vano largo m. 0.90 ed alto m. 1.70. A m. 1 dalla descritta parete ve ne è un'altra della medesima costruzione, ma le dimensioni variano, solamente la larghezza di un blocchetto che è di m. 0.62 si è potuto pigliare perché gli altri erano tagliati da un cunicolo. Le pareti così disposte fanno pensare che era un corridoio che mena nella cucina, la quale è nel piano ove vi sono le pitture.

\section{Pièces 21-22}

28 febbraio 1933. Sul solaio del piano superiore interno, non ancora enumerato, della casa $\mathrm{N}^{\circ} 6$, sul IV cardine, lato est, a m. 1.20 dalla parete sud, ed a m. 1.75 da quella ovest si è raccolto: [Description des objets inventoriés 1031 à 938 et de la base d'un vase de céramique arétine marqué]. ${ }^{63}$

10 marzo 1933. Nella casa $\mathrm{N}^{\circ} 6$ sul solarino del piano superiore a m. 1.27 dalla parete ovest ed a m. 0.70 da quella nord è stato raccolto: [Description des objets inventoriés 1046 à 1047].64

[Description finale (insérée après juin 1933)] Ambiente n. 6 [= pièce 22]. [...] L'ambiente è di forma rettangolare e misura m. 2.52 di lunghezza per m. 3.64 di larghezza mentre l'altezza è di m. 2.70. Il solarino del piano superiore è sostenuto da otto travi di legno a forma e dimensioni differenti. [...] Al piano superiore vi si saliva a mezzo della scala di legno posta nell'ambiente $n$. 2. Le pareti del secondo piano, quella nord ed est conservano buona parte della decorazione che è a fondo bianco la parte alta e di rosso e bianco quella bassa. Sul fondo rosso, parete nord, vi è un quadretto con un bel paesaggio festoni di fiori 
e qualche vaso. Sui riquadri laterali, bianco, ippocampi sopra prospettive e festoni di foglie. [...] Il vano d'ingresso del piano superiore è nell'angolo sud ovest. [...]

\section{Objets découverts dans les pièces 21-22}

1031 [= 76308] (23/02/1933) : Bronzo. Ornamento di spalliera di letto? È una donna seduta che stringe con la mano sinistra una lira? e con la destra si accinge a suonare. L'altezza è di m. 0.08 . $1032[=76309](23 / 02 / 1933):$ Terracotta. Lucerna monolichne col fondo superiore rotto. Misura m. 0.025 di altezza, m. 0.08 di lunghezza e m. 0.04 di diametro. Inventario n. 1032. $1046[=76323](10 / 03 / 1933):$ : Bronzo. Circolo del diametro di m. 0.32 ed alto m. 0.08 . 1047 [= 76324] (10/03/1933) : Bronzo. Moneta di modulo medio del diametro di m. 0.027 . Sans inventaire $(28 / 02 / 1933):[. .$.$] un fondo di vaso di terracotta aretina che tiene il$ seguente bollo sul fondo superiore: [dessin d'un timbre C.MVRRI in planta pedis dextrorsum 65]. È lungo m. 0.023 ed alto m. 0.003 .

\section{Pièce 23}

25 aprile 1933. Sul piano superiore del tablino della casa $\mathrm{N}^{\circ} 6,4^{\circ}$ cardine, lato est, a m. 1.15 dalla parete sud ed a m. 0.40 dall'estremità di quella ovest è stato raccolto: [Description des objets inventoriés 1074 à 1075].

5 maggio 1933. Nella casa n. 6 sul piano superiore del tablino sopra il secondo pilastro, lato Nord vi sono moltissimi graffiti, essi sono: [Transcription des graffiti CIL IV, 10565-1056766].

[Description finale (insérée après juin 1933)] Ambiente $\mathrm{n} .9$ [tablinum 3, en dessous de la pièce 23]. [...] Sopra il tablino vi è il piano superiore, il cui battuto è sostenuto da 7 travicelli a fondo rettangolare. L'ambiente è alto m. 3.42. [...]

Ambiente n. 13 [nymphée 8]. [...] Nessuna traccia di scale si è trovato per salire al piano superiore che trovasi sul tablino, ma a mio parere, unico mezzo era quello di una scala di legno portatile, tanto più che detto ambiente era un ripostiglio, e ciò lo fa supporre una lunga serie di graffiti che sono sopra un pilastro di vano che ci fa nota la consegna di utensili che il proprietario da al proprio garzone. I graffiti sono quelli che seguono: [Transcription des graffiti CIL IV, 10565-10567] [...]

\section{Objets découverts dans la pièce 23}

1074 [=76351] (25/04/1933): Bronzo. Lucerna monolichne e monoansata con una palmetta all'estremità posteriore. Il rostro è prolungato ed il piede è sollevato. Misura m. 0.11 di lunghezza per m. 0.022 di diametro. Il piede è alto m. 0.005 .

1075 [= 76352] (25/04/1933) : Bronzo. Lucerna con rostro prolungato e piede sollevato. Era tenuta sospesa da due catiniglie di bronzo. La lunghezza è di m. 0.14 ed il diametro di m. 0.03. 


\section{BIBLIOGRAPHY}

Andrews $2006=\mathrm{J} . \mathrm{N}$. Andrews, The use and development of upper floors in houses at Herculaneum, PhD thesis, University of Reading, 2006.

Andrews $2017=$ J.N. Andrews, Revisiting the upper floors of the Casa d'Argo at Herculaneum, dans Anabases, 117, 2017, p. 117-141.

Barbet 1981 = A. Barbet, Les bordures ajourées dans le IV style de Pompéi. Essai de typologie, dans MEFRA, 93-2, 1981, p. 917-998.

Bastet - De Vos 1979 = F.-L. Bastet, M. De Vos, Proposta per una classificazione del terzo stile pompeiano, 's-Gravenhage, 1979 (Archeologische studien van het Nederlands historisch instituut te Rome ,4).

Bechi 1831 = G. Bechi, Funamboli - Antichi dipinti di Pompei [tav. LI-LII], dans Real museo Borbonico, 7, 1831, p. 1-8.

Borgongino 2006 = M. Borgongino, Archeobotanica. Reperti vegetali da Pompei e dal territorio vesuviano, Rome, 2006 (Studi della Soprintendenza archeologica di Pompei, 16).

Camardo - Notomista 2017 = D. Camardo, M. Notomista, Ercolano: 1927-1961. L'impresa archeologica di Amedeo Maiuri e l'esperimento della città museo, Rome, 2017 (Studi e Ricerche del Parco Archeologico di Pompei, 34).

Catalano 2002 = V. Catalano, Case, abitanti e culti di Ercolano, Rome, 2002.

Dardenay et al. 2015 = A. Dardenay, A. Allroggen-Bedel, H. Eristov, M.-L. Maraval, N. Monteix, Habitat et société à Herculanum, dans Chronique des activités archéologiques de l'École française de Rome, 2015, consulté le 15 juin 2018 : https://journals.openedition.org/cefr/1339.

Dardenay et al. 2016 = A. Dardenay, A. Allroggen-Bedel, H. Eristov, A. Grand-Clément, M.-

L. Maraval, C. Marotta, N. Monteix, E. Rosso, Herculanum. Des archives aux restitutions architecturales et décoratives, dans Chronique des activités archéologiques de l'École française de Rome, 2016, consulté le 15 juin 2018 : https://journals.openedition.org/cefr/1588.

Della Corte 1958 = M. Della Corte, Le iscrizioni di Ercolano, dans RAAN, 33, 1958, p. 239-308.

Eristov 1994 = H. Eristov, Les éléments architecturaux dans la peinture campanienne du quatrième style, Rome, 1994 (Collection de l'École française de Rome, 187).

Esposito 2014 = D. Esposito, La pittura di Ercolano, Rome, 2014 (Studi della Soprintendenza archeologica di Pompei, 33).

Guidobaldi et al. 2014 = F. Guidobaldi, M. Grandi, M.S. Pisapia, R. Balzanetti, A. Bigliati, Mosaici antichi in Italia. Regione prima. Ercolano, Pise-Rome, 2014.

Helbig 1868 = W. Helbig, Wandgemälde der vom Vesuv verschütteten Städte Campaniens, Leipzig, 1868.

Maiuri 1958 = A. Maiuri, Ercolano. I nuovi scavi (1927-1958), Rome, 1958.

Mols 1999 = S.T.A.M. Mols, Wooden furniture in Herculaneum: form, technique and function, Amsterdam, 1999 (Circumvesuviana, 2). 
Monteix 2010 = N. Monteix, Les lieux de métier. Boutiques et ateliers d'Herculanum, Rome, 2010 (BEFAR , 344).

Orr 1972 = D.G. Orr, Roman domestic religion: $a$ study of the Roman household deities and their shrines at Pompeii and Herculaneum, PhD Thesis, University of Maryland, 1972.

Oxé - Comfort - Kenrick $2000=$ A. Oxé, H. Comfort, P. Kenrick, Corpus vasorum Arretinorum: $a$ catalogue of the signatures, shapes and chronology of Italian Sigillata, Bonn, 2000, $2^{\mathrm{e}}$ éd. (Antiquitas. Reihe 3, 41).

Reinach 1970 = S. Reinach, Répertoire de peintures grecques et romaines, Rome, 1970 .

Strocka 1975 = V.M. Strocka, Pompejanische Nebenzimmer, dans B. Andreae, H. Kyrieleis (éd.), Neue Forschungen in Pompeji und den anderen vom Vesuvausbruch 79 n. Chr. verschütteten Städten, Recklinghausen, 1975, p. 101-114.

Tassinari 1993 = S. Tassinari, Il vasellame bronzeo di Pompei, Rome, 1993 (Cataloghi della Soprintendenza archeologica di Pompei, 5).

Varone 2012 = A. Varone, Titulorum graphio exaratorum qui in C.I.L. Vol. IV collecti sunt imagines, Rome, 2012 (Studi della Soprintendenza archeologica di Pompei, 31).

Wallace-Hadrill 1994 = A. Wallace-Hadrill, Houses and society in Pompeii and Herculaneum, Princeton, 1994.

\section{NOTES}

1. Cette étude de l'étage se fonde sur les travaux de J. Andrews (2006), collaborateur du projet, revus avec le reste de l'équipe.

2. Guidobaldi et al. 2014.

3. Guidobaldi et al. 2014, p. 22 pour la justification du mode de datation des pavements.

4. En raison de la pente prononcée du sol des fauces, cette mesure est prise à mi-longueur de la pièce.

5. Le dénivelé est de 15,5 cm pour une longueur de $4 \mathrm{~m}$ (soit un pendage d'un peu moins de $4 \%$ ).

6. Guidobaldi et al. 2014, $\mathrm{n}^{\circ} 252$, p. 284.

7. Dardenay et al. 2016, p. 16.

8. Guidobaldi et al. 2014, no 93, p. 125-126.

9. Voir, par exemple, à Pompéi, dans la casa di Trebio Valente (III 2,1), l'ala m (Strocka 1975, fig. 82), ou dans la casa dei Vettii (VI 15, 1), le cubiculum $k$ (Strocka 1975, fig. 93).

10. Reinach 1970, 349.2.

11. Helbig $1868, \mathrm{n}^{\circ} 898$.

12. Bechi 1831 , pl. 52, p. 8 .

13. Nous remercions Iris Pingeon de ces précisions.

14. Andrews 2006, vol. 1, p. 63 sq. et table 3.5.

15. Phase Ia (Bastet - De Vos 1979, p. 24 sq.) : Pompéi, casa dei gladiatori (V 5, 3) ; casa del Gallo (VIII 5, 2).

16. Sur les dommages consécutifs au séisme dans la casa di Nettuno ed Anfitrite, Monteix 2010, p. 326-329; Andrews 2006, vol. 1, p. 200 ; sur les modifications architecturales, Andrews 2006, vol. 1, p. 211.

17. Si le plancher de l'étage a été refait, cette restructuration prendrait place durant cette phase. On peut toutefois supposer que cette réfection date de l'époque moderne et que la réduction de hauteur de la pièce induite par ces travaux relève d'une erreur de restauration.

18. Barbet 1981, p. 966, fig. 17. 
19. Maiuri 1958, p. 401-402, fig. 331, pl. XXXII.

20. Concernant la présentation de la maison au public, voir Camardo - Notomista 2017, p. 53-55, fig. 62.

21. Voir le texte des Giornali degli Scavi (= GSE) infra en annexe. D'après un lambeau d'enduit préservé sur le mur sud, l'escalier montait à un angle d'environ $45^{\circ}$.

22. La " pièce » 17 présente un statut particulier en ce sens que ces limites exactes ne sont pas connues. En l'état actuel, consécutif aux travaux d'A. Maiuri, sa limite occidentale serait donnée par l'extension maximale (inconnue) de l'encorbellement au-dessus du trottoir. Faute de données de fouille, il est impossible de déterminer si, dans l'Antiquité, la pièce 16 continuait jusqu'à la rue en étant percée de deux portes permettant d'atteindre les pièces 14-15 au nord et 18-19 au sud, ou bien s'il existait une pièce 17 véritablement constituée en plan.

23. A. Maiuri (Maiuri 1958, p. 401) indique que la hauteur du plafond à l'étage atteignait 3,53 m. En l'état, la hauteur du plafond des pièces à l'étage a été mesurée à environ 3,60 m. Faute de description dans les GSE antérieure à la restauration, il est difficile d'établir si les piliers maçonnés constituaient des éléments porteurs dans l'Antiquité reconstruits à l'identique ou bien s'il s'agit d'une reconstruction rendue nécessaire par l'écorché de la façade.

24. Des cloisons similaires ont été mises en évidence dans l'appartement (IV,3) de la casa dell'alcova (IV, 4) et dans l'appartement (V, 13-14) de la casa del bicentenario (V, 15-16).

25. D. Esposito (Esposito 2014, p. 89) note la ressemblance entre ces peintures et celles dans deux pièces de l'appartement à l'étage $(\mathrm{V}, 15)$ de la casa del bicentenario, et conclut qu'elles sont probablement issues du même atelier.

26. À propos des traces de rideaux à l'étage de la casa d'Argo (II, 2) et pour une discussion plus détaillée, voir Andrews 2017, p. 135.

27. Maiuri 1958, p. 401-402. À propos des vestiges de la banquette en bois et du lieu de découverte, voir Mols 1999, p. 27, 159-160, 250 (citant les GSE du 9 juin 1933), cat. $n^{\circ}$ 8, fig. 61. En ce qui concerne le catalogue des objets places dans cette pièce après les fouilles, voir Camardo Notomista 2017, p. 206, fig. 1-2.

28. GSE $1933: 1^{\circ}$ maggio, cités infra en annexe. Sur le déplacement des fèves, voir Monteix 2010, p. 22-23.

29. GSE $1932: 1^{\circ}$ novembre, cités infra en annexe.

30. Le laraire ne fait pas partie du catalogue proposé dans Orr 1972.

31. GSE 1932 : 14 novembre ; description finale [insérée après le mois de juin 1934]; cités infra en annexe.

32. Les Lares sont peints en rouge et portaient vraisemblablement une tunique et une coiffe; le Lare de droite est figuré jambes nues. Le Lare de gauche soulève un rhyton dans sa main gauche et tient une situla dans sa main droite, tandis que le Lare de droite tient un rhyton dans sa main droite et une situla dans sa main gauche.

33. Eristov 1994, type II, fig. 43 (= Varano, villa d'Arianna, 5)

34. La position centrée de l'édicule, inséré dans un décor de IV ${ }^{\mathrm{e}}$ style interdit donc que l'espace correspondant à l'encorbellement ait revêtu la même décoration. Deux hypothèses concurrentes peuvent être proposées pour expliquer cette situation : soit l'encorbellement correspondait à une pièce indépendante, soit il n'y avait pas d'encorbellement durant cette phase.

35. Andrews 2006, vol. II, p. 189.

36. La partie conservée du siège se situe à une distance de $0,24 \mathrm{~m}$ du mur est et mesure $0,32 \mathrm{~m}$ de large, 0,52 $\mathrm{m}$ de haut et $0,37 \mathrm{~m}$ de profondeur.

37. Dardenay et al. 2015.

38. GSE, description finale [insérée après le mois de juin 1934], cf. annexes, infra. L'aspect de ces échappées devait être proche de celles de la pièce 19 à l'étage, au-dessus de la boutique ; J. Andrews (Andrews 2006, vol. II, p. 187) avait encore pu discerner en bas des échappées une trace de barrière surmontée de « a kind of object » dont il ne reste rien. 
39. GSE, description finale (insérée après le mois de juin 1934), cf. annexes, infra.

40. Voir annexes, infra.

41. Les traces de ces ouvertures sont dissimulées par le rehaussement moderne du mur ouest de la cour : celui-ci dépasse le niveau du sol de l'étage, destiné à supporter la toiture de protection installée après les travaux de restauration.

42. GSE, description finale [insérée après le mois de juin 1934], cf. annexes, infra.

43. GSE 1933 : 15, 31 maggio ; description finale [insérée après le mois de juin 1934], cf. annexes, infra. L'enduit sur lequel les graffiti ont été tracés fut déplacé lors des restaurations sur la partie supérieure du mur ouest de la cour 8 , où ils sont toujours visibles.

44. CIL IV, 10565 : Vinum acceptum / ab domino VII Idus Apriles. Voir également Della Corte 1958, p. 270-271, no 387, pl. III ; Catalano 2002, p. 65, pl. XXXV.2-3 ; Varone 2012, p. 492 (G/594).

45. CIL IV, 10566 : Aquaria dua cum basis / aqua in manus dua cum basis / hamas duas cum basis / aqua in manu cotidian CII / cum basis / urciolus duos / candelabra quat $(t)$ uor / et lucubratoriu $(m)$ unum / lucerna aenea / hamula una / pelvi(s) cum basim / et lytrum / gut(t)os tres / scapheola dua / ferreas strig(i)les VII | \{h\}aenas quattuor || marmor cum basim | aenea | fuminaria dua. Voir également Della Corte 1958, p. 271, n 388, pl. III ; Catalano 2002, p. 65, pl. XXXV.2-3 ; Varone 2012, p. 492 (G/595).

46. CIL IV, 10567 : Branc | broc | trans | mus || Nos | ter | tros | men || Quod | quid | quae | quas || Rum | quis | que | dem || Con / les / gis | mul || Mol | mae | me | mae. Voir également Della Corte 1958, p. 271, n 389, pl. III ; Catalano 2002, p. 65, pl. XXXV.4; Varone 2012, p. 493 (G/596). V.Catalano (Catalano 2002, p. 64) suggère que cet exercice grammatical pourrait être le résultat d'un travail d'un ludimagister, un maître d'école responsable de l'éducation de jeunes enfants. Eu égard à la difficulté d'accès du lieu, cette interprétation semble assez improbable.

47. Le terme d'aquum in manus cum basis, éventuellement d'usage "quotidien " (et alors au nombre de 102 ?), paraît également renvoyer vers le service de l'eau. Il pourrait s'agir de récipients de petites dimensions, devant (?) être tenus « en main », parce que dépourvus d'anse.

48. Les sept strigiles en fer sont d'interprétation plus délicate: il pourrait s'agir de strigiles confiés au personnel servile pour qu'il puisse les utiliser pour son usage, ce qui pourrait permettre d'évaluer le nombre d'esclaves présents dans cette maison à (un maximum de ?) sept ; on préfèrera cependant une autre interprétation, qui fait de ces strigiles des instruments confiés au personnel servile pour qu'il s'occupe de son maître aux thermes.

49. Wallace-Hadrill 1994, p. 116.

50. Concernant une discussion récente sur la possible utilisation saisonnière des étages dans la casa d'Argo (II, 2), voir Andrews 2017, p. 139-140.

51. Dans le volume relié conservé à Herculanum, le texte est inséré après le résumé mensuel des travaux effectués en juin 1934.

52. Le lieu de fouille n'est pas précisé. Quatre jours auparavant (25 avril 1933), la fouille se déroule à l'étage de la pièce 23, mais le jour ouvré suivant (lundi $1^{\mathrm{er}}$ mai 1933), c'est la façade extérieure qui est fouillée, renvoyant ainsi plutôt à la pièce 14 .

53. Lu comme [T]igilli Iuli[ae] et indiqué par erreur comme provenant de IV, 4 (Della Corte 1958, n o 343 p. 268).

54. CIL IV, 10755. Lu Oleti et indiqué par erreur comme provenant de IV, 4 (Della Corte 1958, $\mathrm{n}^{\circ}$ 345 p. 268), repris par P. Ciprotti dans le CIL.

55. Comme on ne compte que 13 pièces au rez-de-chaussée, il est possible que la pièce « ambiente $\mathrm{n}^{\circ} 16$ » renvoie à l'étage. Toutefois, l'objet est donné comme ayant été découvert «a m. 0.37 di altezza dal suolo del pavimento ", qui renvoie plus vers le rez-de-chaussée que vers l'étage pour lequel solarino est plus généralement utilisé. Cette pièce n'est jamais mentionnée ailleurs dans les GSE ou dans le Giornale dei lavori eseguiti (GL) qui reprend notamment les restaurations effectuées, ce qui rend sa localisation impossible. La provenance indiquée dans les livres d'inventaire n'est guère plus précise, renvoyant initialement de manière générique à la « Casa N. $6-4^{\circ}$ cardine lato est ».

Chronique des activités archéologiques de l'École française de Rome, Les cités vésuviennes 
56. Il est probable que le lieu de découverte de ces objets soit plutôt les pièces 14 ou 15 que le couloir 17 situé en face de la pièce 16. En effet, la localisation du coffre en bois et des amphores est relativement précise : le premier a été observé à proximité du mur nord et les secondes à proximité des murs nord et est. Comme le couloir 17 ne dispose ni de mur au nord (où il s'ouvre sur la pièce 15) ou à l'est (où il s'ouvre sur les pièces 14 et 16), il peut être exclu. Il apparaît plus vraisemblable que le coffre et les amphores aient été découverts dans la pièce 15 située plus vers la rue que la pièce 14 et donc susceptible d'avoir été dégagée en premier.

57. Cette amphore pourrait provenir soit de la pièce 15 , soit, plus vraisemblablement de la pièce 14 qui n'aurait été que partiellement dégagée en novembre 1932. La description tardive des pièces 1 et 2 (respectivement le 14 juin 1933 et le 15 mai 1933), situées au rez-de-chaussée sous les pièces 14 et 15 , tendrait à accréditer l'hypothèse d'une fouille seulement partielle de ces deux pièces jusqu'en mai 1933.

58. Sur le fond de la casserole est attaché par la corrosion le fond d'un récipient en forme de coquille (Tassinari 1993, p. 81-82 [type N], cf. aussi p. 232 pour la fonction), fréquemment appelé «forme di pasticceria ». Les deux objets ont un seul numéro d'inventaire.

59. Lu comme Hebuna et indiqué par erreur comme provenant de IV, 17 (Della Corte 1958, $\mathrm{n}^{\circ}$ 337 p. 267).

60. Borgongino 2006, p. 80.

61. Borgongino 2006, p. 121.

62. CIL IV, 10777. Lu ISVCR / XXIV et indiqué par erreur comme provenant de IV, 4 (Della Corte 1958, n 381 p. 270), repris par P. Ciprotti dans le CIL. De manière assez étonnante, les fèves contenues dans cette amphore n'ont pas été inventoriées. Elles pourraient avoir été déposées dans les dolia de la boutique $\mathrm{V}, 6$.

63. Il est vraisemblable que l'adjectif interno renvoie aux pièces de l'étage donnant sur l'atrium (pièces 20 et 21 ). Comme la pièce 21 est principalement occupée par la partie sommitale de l'escalier d'accès, elle peut probablement être écartée, ce qui donnerait la pièce 22 comme lieu de découverte de ces objets.

64. Le lieu de découverte est incertain. Cependant, comme les pièces 23 et 24 sont clairement indiquées dans le mois suivant (avril), il est possible que cette description renvoie aux pièces 21 ou 22.

65. L'apographe reporté dans les journaux de fouille permet de restituer un timbre OCK type 1203, sans qu'il ne soit possible de choisir entre les 11 variantes attestées faute de pouvoir effectuer une nouvelle autopsie (OCK, 1203.21-32, voir Oxé - Comfort - Kenrick 2000, p. 289).

66. Cit. supra n. 44-46.

\section{INDEX}

institutions Parco Archeologico di Ercolano, Museo Archeologico Nazionale di Napoli, École française de Rome, Centre Jean Bérard, CNRS, ANR, ENS, université Toulouse Jean Jaurès, Laboratoire TRACES (UMR 5608). 


\section{AUTHORS}

\section{ALEXANDRA DARDENAY}

Université Toulouse II Jean Jaurès, UMR 5608 « TRACES » (CNRS/université de

Toulouse II) - adardenay[at]yahoo.fr

JAMES N. ANDREWS

Independent researcher, jamesnandrews@outlook.com

\section{HÉLÈNE ERISTOV}

UMR 8546 « AOROC » (CNRS/ENS) - helene.eristov[at]ens.fr

\section{MARIE-LAURE MARAVAL}

Université Toulouse II Le Mirail, DAR (Direction Appui Recherche), UMR 5608 « TRACES » (CNRS/ université de Toulouse II) - mmaraval[at]univ-tlse2.fr

\section{NICOLAS MONTEIX}

Institut universitaire de France/université de Rouen, GRHis (EA 3831) - nicolas.monteix[at]univrouen.fr 